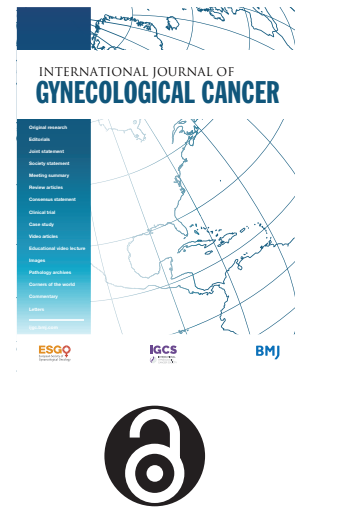

\section{OPEN ACCESS}

- Additional supplemental material is published online only. To view, please visit the journal online (http://dx.doi.org/ 10.1136/ijgc-2021-002565).

For numbered affiliations see end of article.

Correspondence to Professor Dirk Timmerman, Gynecology and Obstetrics, University Hospitals KU Leuven, Leuven, Belgium; Dirk. timmerman@uzleuven.be

For 'Presented at statement' see end of article.

Received 25 February 2021 Accepted 8 March 2021 Published Online First 10 June 2021

\title{
ESGO/ISUOG/IOTA/ESGE Consensus Statement on pre-operative diagnosis of ovarian tumors
}

\begin{abstract}
Dirk Timmerman (D) , , ${ }^{1,2}$ François Planchamp, ${ }^{3}$ Tom Bourne (D) , ${ }^{1,2,4}$ Chiara Landolfo (D) , Andreas du Bois, ${ }^{6}$ Luis Chiva (D) , David Cibula (D) , ${ }^{8}$ Nicole Concin (D) , ${ }^{6,9}$ Daniela Fischerova (D) , Wouter Froyman (1D) , Guillermo Gallardo Madueño (D) , ${ }^{10}$ Birthe Lemley, ${ }^{11,12}$ Annika Loft, ${ }^{13}$ Liliana Mereu, ${ }^{14}$ Philippe Morice, ${ }^{15}$ Denis Querleu (1) , 16,17 Antonia Carla Testa (D) , 5,18 Ignace Vergote, ${ }^{19}$ Vincent Vandecaveye (D) , ${ }^{20,21}$ Giovanni Scambia,,${ }^{5,18}$ Christina Fotopoulou (i) 22
\end{abstract}

\begin{abstract}
The European Society of Gynaecological Oncology (ESGO), the International Society of Ultrasound in Obstetrics and Gynecology (ISUOG), the International Ovarian Tumour Analysis (IOTA) group, and the European Society for Gynaecological Endoscopy (ESGE) jointly developed clinically relevant and evidence-based statements on the pre-operative diagnosis of ovarian tumors, including imaging techniques, biomarkers, and prediction models. ESGO/ISUOG/IOTA/ESGE nominated a multidisciplinary international group, including expert practising clinicians and researchers who have demonstrated leadership and expertise in the pre-operative diagnosis of ovarian tumors and management of patients with ovarian cancer (19 experts across Europe). A patient representative was also included in the group. To ensure that the statements were evidence-based, the current literature was reviewed and critically appraised. Preliminary statements were drafted based on the review of the relevant literature. During a conference call, the whole group discussed each preliminary statement and a first round of voting was carried out. Statements were removed when a consensus among group members was not obtained. The voters had the opportunity to provide comments/suggestions with their votes. The statements were then revised accordingly. Another round of voting was carried out according to the same rules to allow the whole group to evaluate the revised version of the statements. The group achieved consensus on 18 statements. This Consensus Statement presents these ESGO/ISUOG/IOTA/ESGE statements on the pre-operative diagnosis of ovarian tumors and the assessment of carcinomatosis, together with a summary of the evidence supporting each statement.
\end{abstract}

\section{INTRODUCTION}

The accurate characterization of newly diagnosed adnexal lesions is of paramount importance to define appropriate treatment pathways. Patients with masses that are suspicious for malignancy should be referred to a gynecological oncology center, in order to receive specialist care, as per the definitions of the European Society of Gynaecological Oncology (ESGO) ${ }^{1}$ and national and international recommendations and guidelines. For a non-gynecological primary tumor, patients need to be referred to an appropriate specialist, while patients with benign lesions may be followed up and treated conservatively or may be suitable for less radical surgical treatment, depending on the clinical context. ${ }^{2-7}$ Treatment decision-making processes should be based on a combination of the patient's overall clinical picture, symptoms, preferences, previous medical and surgical history, tumor markers, and clinical and radiological findings. A single diagnostic modality alone should not determine the patient's journey.

The ESGO, the International Society of Ultrasound in Obstetrics and Gynecology (ISUOG), the International Ovarian Tumour Analysis group (IOTA), and the European Society for Gynaecological Endoscopy (ESGE) have, jointly, developed clinically relevant and evidence-based statements on the pre-operative diagnosis of ovarian tumors and assessment of disease spread, including imaging techniques, biomarkers, and predictive models. Neither screening and follow-up modalities nor economic analysis of the imaging techniques, biomarkers, and prediction models addressed herein are included within the remit of this Consensus Statement.

\section{RESPONSIBILITIES}

The present series of statements form a consensus of the authors regarding their currently accepted approaches for the pre-operative diagnosis of ovarian tumors and assessment of disease spread, based on the available literature and evidence. Any clinician applying or consulting these statements is expected to use independent medical judgment in the context of individual clinical circumstances to determine all patients' care and treatment. These statements are presented without any warranty regarding their content, use or application, and the authors disclaim any responsibility for their application or use in any way.

\section{METHODS}

This Consensus Statement on the pre-operative diagnosis of ovarian tumors and assessment of disease spread was developed using an eight-step process, chaired by Professors Christina Fotopoulou and Dirk 


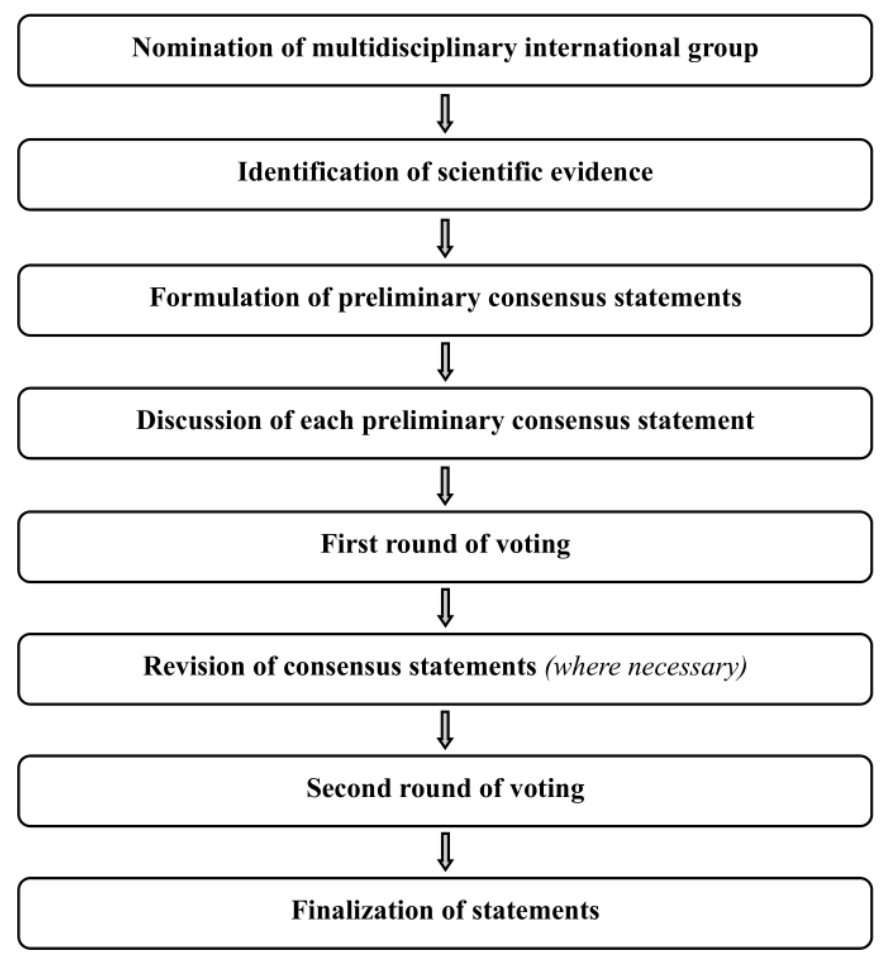

Figure 1 Eight-step process for development of the Consensus Statement on the pre-operative diagnosis of ovarian tumors and assessment of disease spread.

Timmerman (Figure 1). Aiming to assemble a multidisciplinary international group, ESGO/ISUOG/IOTA/ESGE nominated 19 practising clinicians and researchers who have demonstrated leadership and expertise in the pre-operative diagnosis of ovarian tumors and clinical management of patients with ovarian cancer through research, administrative responsibilities, and/or committee membership (including eight members of ESGO, five members of ISUOG, four members of IOTA, and two members of ESGE). These experts included seven gynecologists with special interest in ultrasonography, two radiologists, and 10 gynecological oncologists. They did not represent the societies from which they were selected, and were asked to base their decisions on their own experience and expertise. Also included in the group was a patient representative, who is Chair of the Clinical Trial Project of the European Network of Gynaecological Cancer Advocacy Groups (ENGAGe). An initial conference call including the whole group was held to facilitate introductions, as well as to review the purpose and scope of this Consensus Statement.

To ensure that the statements were evidence-based, the current literature was reviewed and critically appraised. Thus, a systematic literature review of relevant studies published between 1 May 2015 and 1 May 2020 was carried out using the MEDLINE database (Online supplemental appendix 1). The literature search was limited to publications in the English language. Priority was given to highquality systematic reviews, meta-analyses, and validating cohort studies, although studies with lower levels of evidence were also evaluated. The search strategy excluded editorials, letters, and case reports. The reference list of each identified article was reviewed for other potentially relevant articles. Final results of the literature search were distributed to the whole group, including electronic full-text versions of each article. One of the authors (FP) provided the methodology and medical writing support for the entire process and did not participate in voting for statements.

The chairs were responsible for drafting preliminary statements based on the review of the relevant literature. These were then sent to the multidisciplinary international group prior to a second conference call. During this conference call, the whole group discussed each preliminary statement and a first round of binary voting (agree/disagree) was carried out for each potential statement. All 20 participants took part in each vote, but they were permitted to abstain from voting if they felt they had insufficient expertise to agree/disagree with the statement or if they had a conflict of interest that could be considered to influence their vote. Statements were removed when a consensus among group members was not obtained. The voters had the opportunity to provide comments/ suggestions with their votes. The chairs then discussed the results of this first round of voting and revised the statements if necessary. The voting results and the revised version of the statements were again sent to the whole group and another round of binary voting was organized, according to the same rules, to allow the whole group to evaluate the revised version of the statements. The statements were finalized based on the results of this second round of voting. The group achieved consensus on 18 statements. In this Consensus Statement, we present a summary of the supporting evidence, the finalized series of statements, and their levels of evidence and grades.

\section{RESULTS}

\section{General remarks}

Even though the test performance of any biochemical or radiological diagnostic test appears to increase after excluding borderline ovarian tumors and non-gynecological primary tumors, such as of the gastrointestinal tract or breast, we included in our literature assessment studies addressing all types of adnexal tumor, as this is a better reflection of clinical reality.

\section{Ultrasonography}

A transvaginal ultrasound examination is often regarded in clinical practice as the standard first-line imaging investigation for the assessment of adnexal pathology. ${ }^{8-11}$ The diagnostic accuracy of ultrasonography in differentiating between benign and malignant adnexal masses has been shown to relate to the expertise of the operator. ${ }^{12-14}$ The European Federation of Societies for Ultrasound in Medicine and Biology has published minimum training requirements for gynecological ultrasound practice in Europe, including standards for theoretical knowledge and practical skills. ${ }^{15}$ These identify three levels of training and expertise. Thus, Level III (expert) can be attributed to a practitioner who is likely to spend the majority of their time undertaking gynecological ultrasound and/or teaching, research and development in the field. A Level II practitioner should have undertaken at least 2000 gynecological ultrasound examinations. The training required to attain this level of practice would usually be gained during a period of expert ultrasound training, which may be within, or after completion of, a specialist training program. To maintain competence at Level II, practitioners should perform at least 500 examinations each year. A Level I practitioner should have performed a minimum of 300 examinations under the supervision of a Level II practitioner or an experienced Level 
I practitioner with at least 2 years' regular practical experience. To maintain Level I status, the practitioner should perform at least 300 examinations each year. A prospective randomized controlled trial to assess the effect of the quality of gynecological ultrasonography on the management of patients with suspected ovarian cancer has shown that women with a Level III (expert) ultrasound examination undergo significantly fewer unnecessary major procedures and have a shorter inpatient hospital stay compared with those having a Level II (routine) examination by a sonographer. ${ }^{9}$

Subjective assessment by expert ultrasound examiners has excellent performance to distinguish between benign and malignant ovarian tumors. ${ }^{9-14}$ In many cases, expert examiners should be able to narrow the diagnosis down further to a specific histological sub-type. The typical pathognomonic ultrasound features of some key histological types have been published in the series 'Imaging in gynecological disease' in Ultrasound in Obstetrics and Gynecology (https://obgyn.onlinelibrary.wiley.com/doi/toc/10. 1002/(ISSN)1469-0705.IMAGINGINGYNECOLOGICALDISEASE) . The most common and typical findings for each pathology are summarized in Table 1.

Risk of malignancy index (RMI) and risk of ovarian malignancy algorithm (ROMA)

Several attempts have been made to develop more objective ultrasound-based approaches for discriminating between benign and malignant adnexal tumors. These include the risk of malignancy index (RMI), a scoring system based on menopausal status, a transvaginal ultrasound score, and serum cancer antigen 125 (CA 125) level. ${ }^{16}$ Many studies have demonstrated the diagnostic performance of the RMI in classifying adnexal masses. ${ }^{117-29}$ Three variants of the RMI (RMI-II, RMI-III, RMI-IV) have been developed, but these offer no significant additional diagnostic advantage compared with the original version (RMI-I). ${ }^{1122}{ }^{27}{ }^{28}$ Moore et a ${ }^{30}$ developed an algorithm, the risk of ovarian malignancy algorithm (ROMA), based on both CA 125 and human epididymis protein 4 (HE4). Westwood et $a^{18}$ pooled data comparing the ROMA with the RMI-I to guide referral decisions for women with suspected ovarian cancer and found similar performance if women with borderline tumors and non-epithelial cancers were excluded from the analyses. More recently, another meta-analysis showed a higher specificity of the RMI-I than the ROMA in pre-menopausal women but a similar performance for detecting ovarian cancer in postmenopausal women presenting with an adnexal mass. ${ }^{17}$ Limitations of the RMI are the absence of an estimated risk of malignancy and its considerable dependency on serum CA 125, the latter resulting in a relatively low sensitivity for early-stage invasive and borderline disease, especially in pre-menopausal women ${ }^{31}{ }^{32}$ (see Tumor Markers).

\section{IOTA methods}

To homogenize and standardize the quality, description, and evaluation of ultrasonography across different centers, and thereby increase diagnostic accuracy, the IOTA group first published a consensus paper on terms and definitions to describe adnexal lesions in $2000 .{ }^{33}$ Using this standardized methodology, the IOTA group has developed different prediction models based on logistic regression analysis. ${ }^{34-36}$ In a large-scale external validation study, Van Holsbeke et $\mathrm{al}^{37}$ showed that the IOTA logistic regression models 1 (LR1, with 12 variables) and 2 (LR2, with six variables) outperformed 12 other models, including the RMI. The LR2 model was easier to use than the LR1 model. Demonstrating the standardization and reproducibility of the IOTA models, Sayasneh et $\mathrm{al}^{38}$ showed that even less experienced sonographers are able to differentiate accurately between benign and malignant ovarian masses using the IOTA LR1 model. The IOTA group also developed 'Simple Rules' that may be applied to a mass based on the presence or absence of five benign and five malignant ultrasound features. These rules can be applied to about $80 \%$ of adnexal masses, with the rest being classed as inconclusive. They have now been broadly accepted and are widely used in clinical practice. ${ }^{38-46}$ More recently, a logistic regression model based on the ultrasound features of the original Simple Rules was developed-the Simple Rules risk model. This model is able to provide an individual estimated risk of malignancy for any type of lesion. ${ }^{35} \mathrm{~A}$ summary of the main models and scoring systems for the pre-operative diagnosis of ovarian tumors is shown in Table 2.

As many ovarian masses can be recognized relatively easily, the IOTA group also proposed four 'Simple Descriptors' of the features typical of common benign lesions and two suggestive of malignancy, which can give an 'instant diagnosis' and reflect the pattern recognition that is a key part of ultrasonography. These are applicable to about $43 \%$ of adnexal masses. ${ }^{47} \mathrm{~A}$ three-step strategy, consisting of the sequential use of Simple Descriptors, Simple Rules, and subjective assessment by an expert, had high accuracy for discriminating between benign and malignant adnexal lesions. ${ }^{47}$ A systematic review and meta-analysis reported better performance of the IOTA Simple Rules and the IOTA LR2 model compared with all other scoring systems, including the RMI. ${ }^{48}$ Besides confirming these findings, another meta-analysis highlighted that a two-step approach, with the IOTA Simple Rules as the first step and subjective assessment by an expert for inconclusive tumors as the second step, matched the test performance of expert ultrasound examiners. ${ }^{11}$ The IOTA Simple Rules have been integrated into several national clinical guidelines for the evaluation and management of adnexal masses, ${ }^{49}{ }^{50}$ and they were considered the main diagnostic strategy ${ }^{51}$ as part of a first international consensus report for the assessment of adnexal masses.

A randomized controlled trial assessing surgical intervention rates and the oncologic safety of decision-making processes using an RMI-based protocol developed by the British Royal College of Obstetricians and Gynaecologists (RCOG) versus triage using the IOTA Simple Rules ${ }^{52}$ showed that the IOTA protocol resulted in lower surgical intervention rates compared with the RMI-based RCOG protocol. The IOTA Simple Rules did not result in more cases in which a diagnosis of cancer was delayed. It was found that the addition of biomarkers such as serum CA 125 and HE4 when using the IOTA Simple Rules, with or without subjective assessment by an expert sonographer, offered no additional diagnostic advantage for the characterization of ovarian masses, but was more costly than a three-step strategy based on the sequential use of the IOTA Simple Descriptors, Simple Rules, and expert evaluation. ${ }^{53} 54$

The IOTA group have also developed the Assessment of Different NEoplasias in the adneXa (ADNEX) model. This multiclass prediction model is the first risk model to differentiate between benign and malignant tumors, while also offering sub-classification of any malignancy into borderline tumors, Stage I, and Stage II-IV primary 


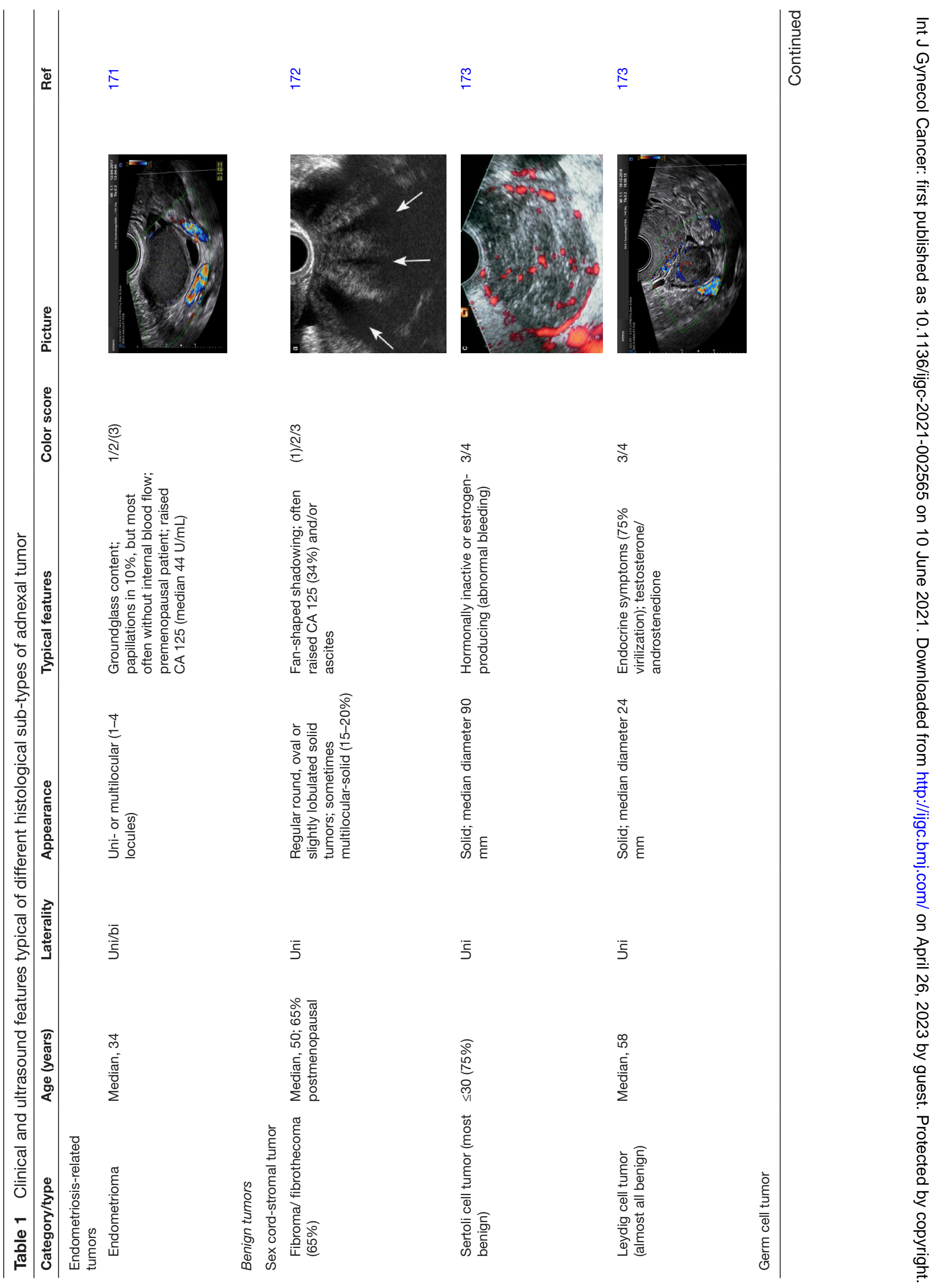


Joint statement

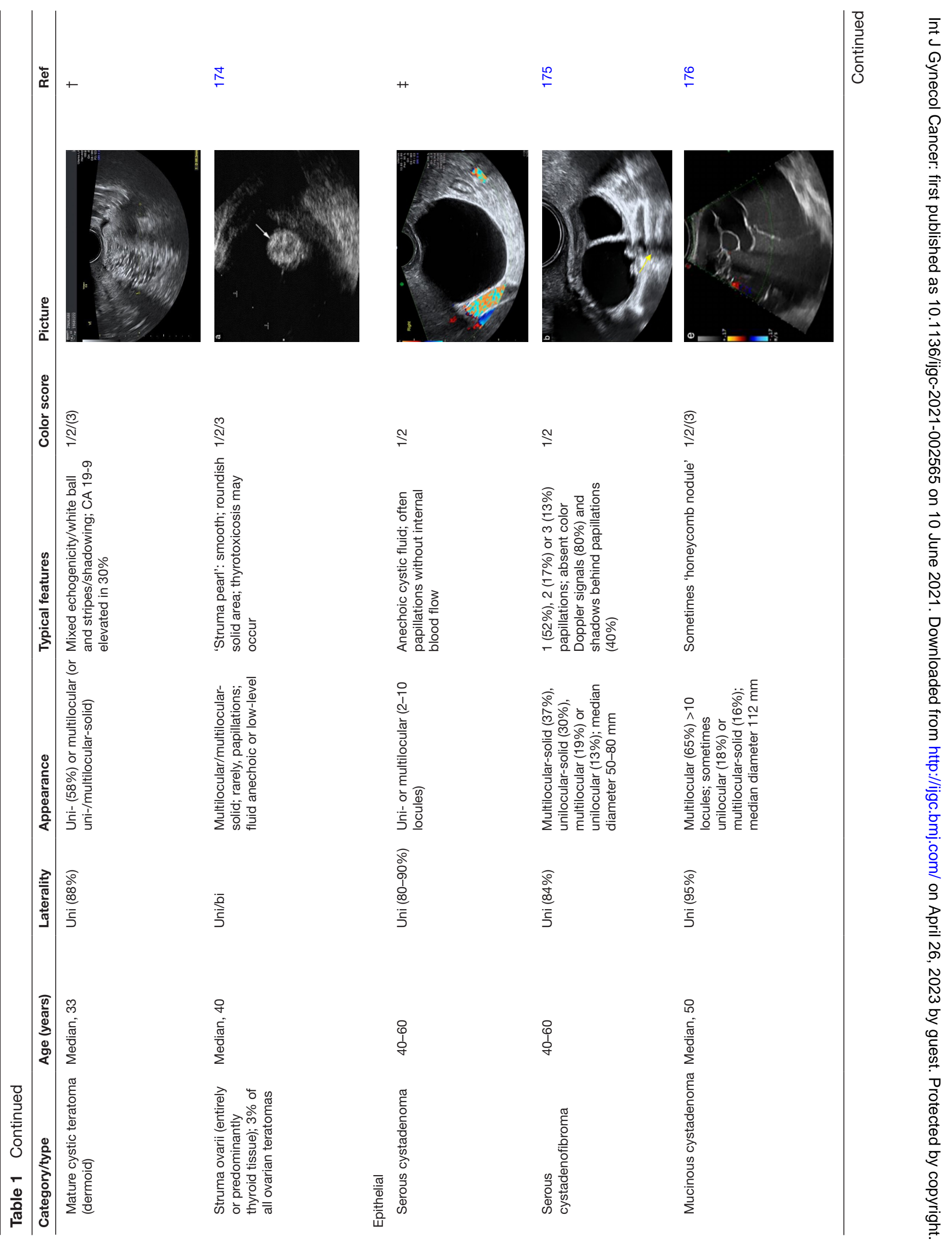




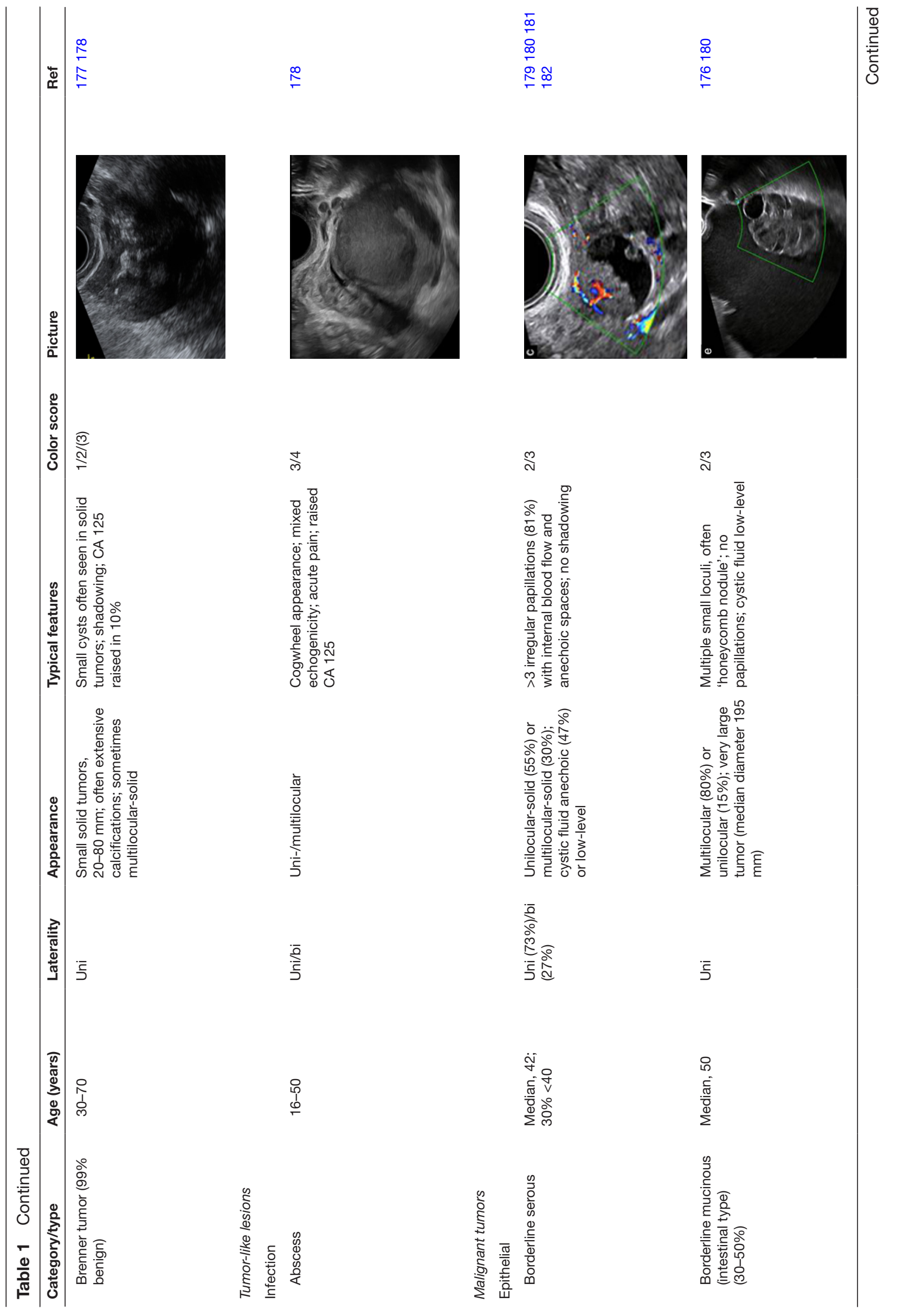


Joint statement

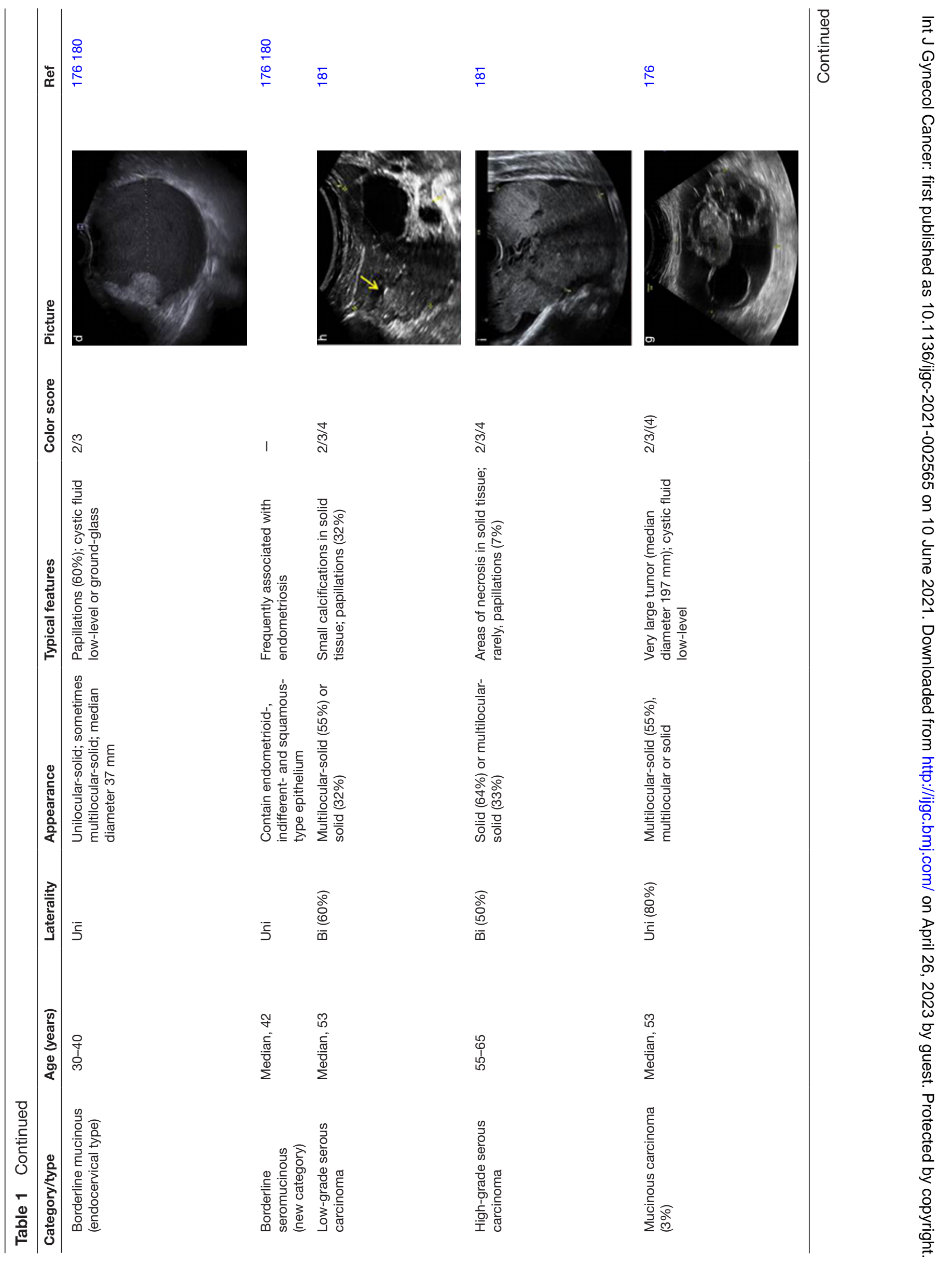




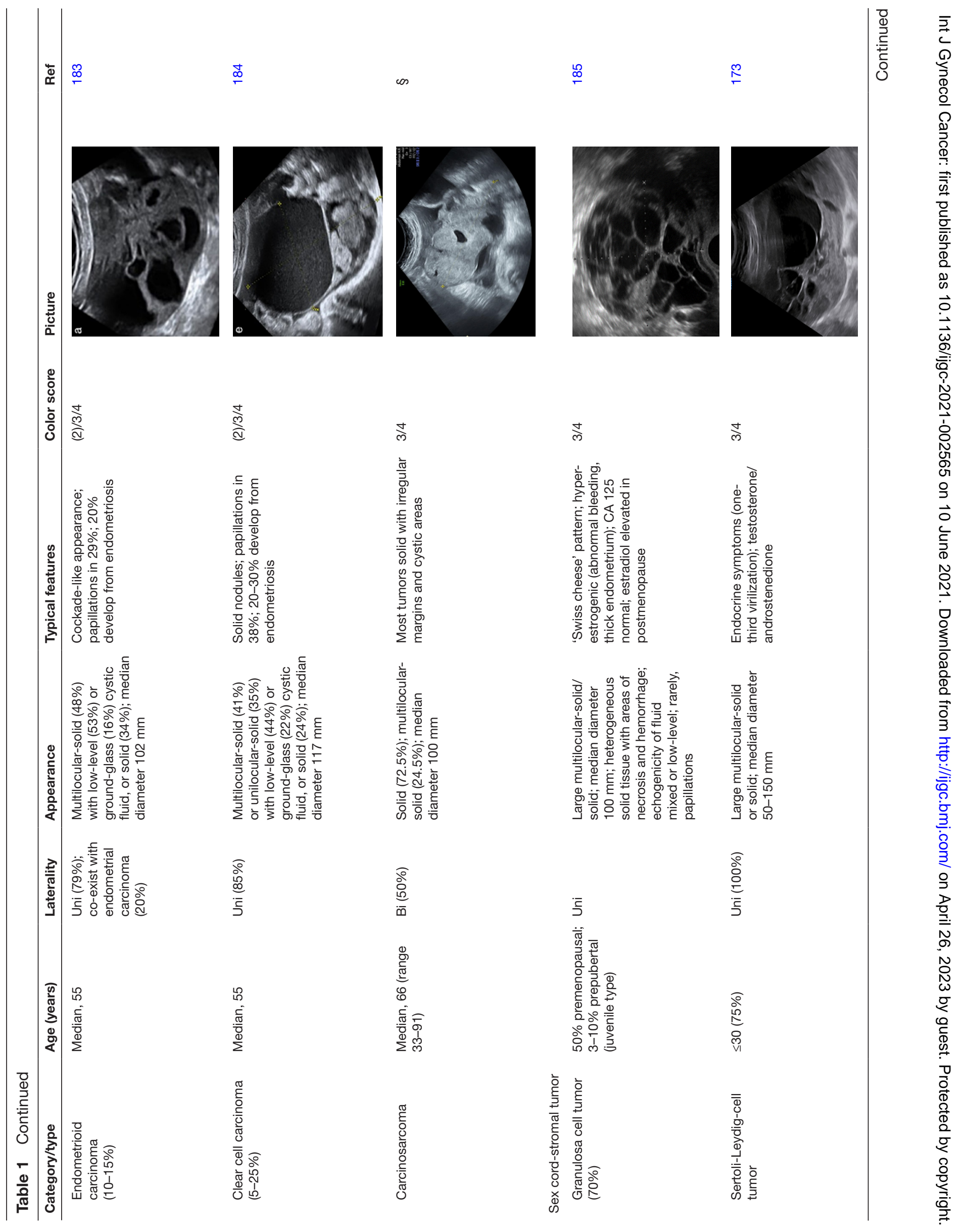


Joint statement

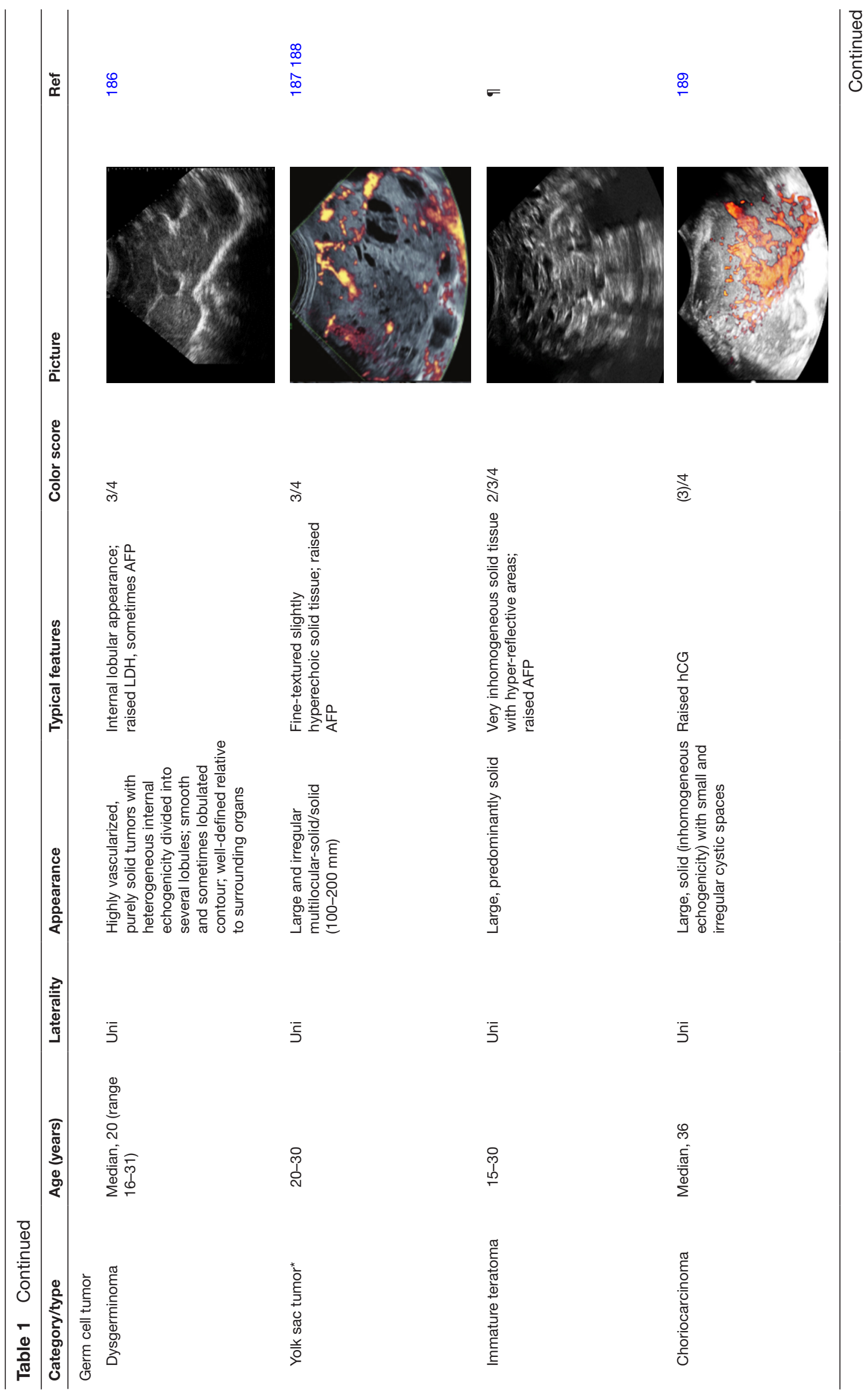

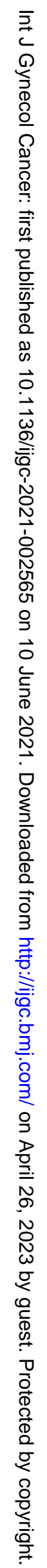




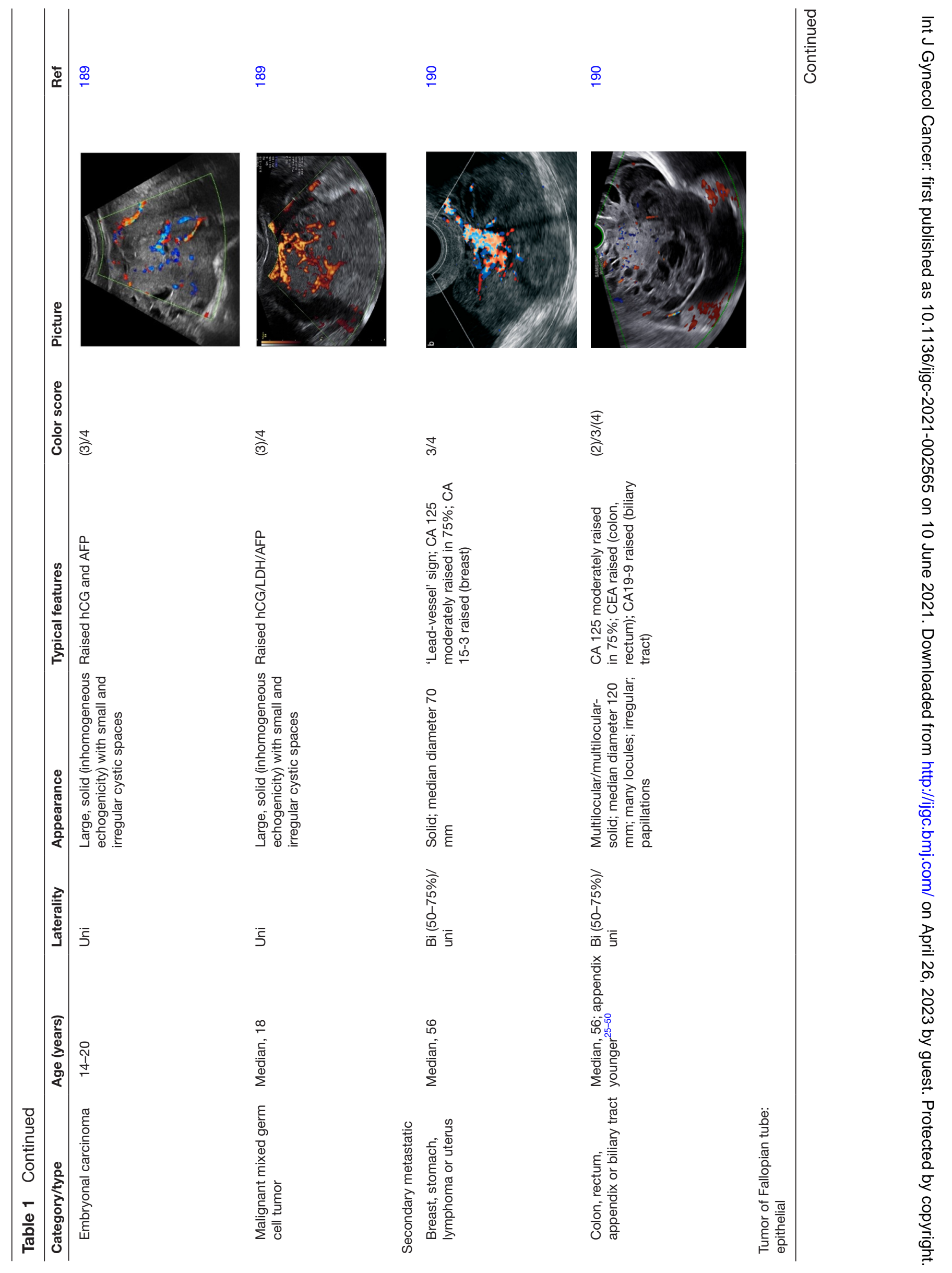




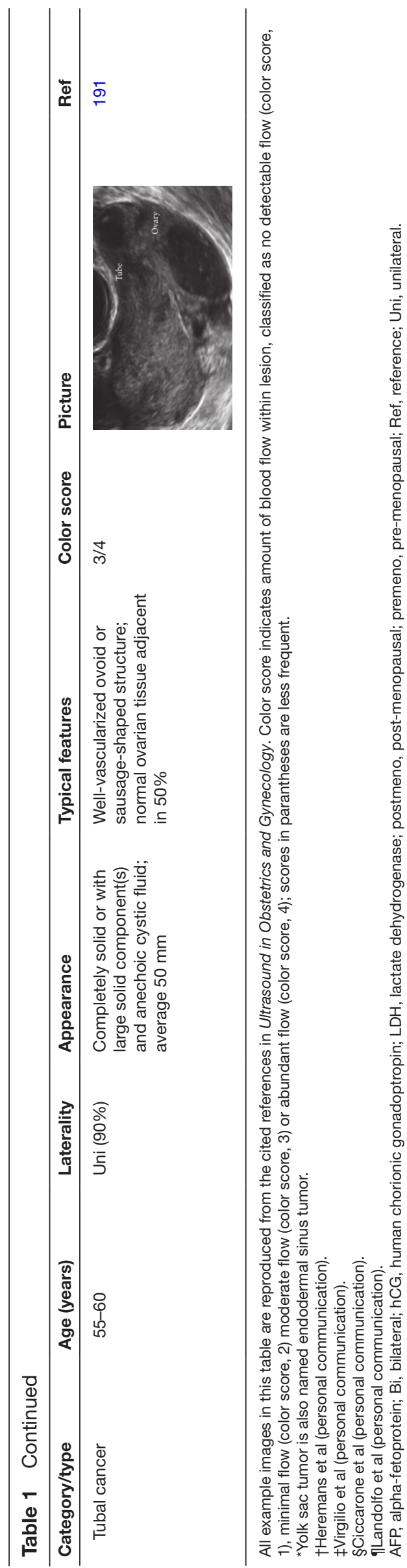

cancers and secondary metastatic tumors. The IOTA ADNEX model was developed and validated using parameters collected by experienced ultrasound examiners. ${ }^{36}$ Several external validation studies have shown good to excellent performance of the ADNEX model in discriminating different types of ovarian tumor, with a higher clinical value than the RMI. ${ }^{55-61} \mathrm{~A}$ study aiming to validate the ADNEX model when applied by Level II examiners has confirmed that it can be used successfully by less-experienced examiners. ${ }^{62} \mathrm{~A}$ large multicenter cohort study of 4905 masses in 17 centers, comparing six different prediction models (RMI, LR2, Simple Rules, Simple Rules risk model, and ADNEX model with or without CA 125), demonstrated the IOTA ADNEX model and the IOTA Simple Rules risk model to be the best models for the characterization of ovarian masses in patients who present with an adnexal lesion. ${ }^{63}$

Gynecologic Imaging Reporting and Data System (GI-RADS)

The Gynecologic Imaging Reporting and Data System (Gl-RADS) was first introduced by Amor et al ${ }^{64}$ in 2009 and was validated prospectively by the same team in a multicenter study 2 years later. ${ }^{65}$ This reporting system quantifies the risk of malignancy into five categories: Gl-RADS 1, definitively benign (estimated probability of malignancy (EPM) $0 \%$ ); GI-RADS 2, very probably benign (EPM <1\%); GI-RADS 3, probably benign (EPM 1-4\%); GI-RADS 4, probably malignant (EPM 5-20\%); and GI-RADS 5, very probably malignant (EPM $>20 \%$ ). More recently, several studies have demonstrated the value of the GI-RADS system for the assessment of malignant adnexal masses in women who are candidates for surgical intervention. Furthermore, the addition of GI-RADS to CA 125 improves the identification of adnexal masses at high risk of malignancy compared with using CA 125 alone. ${ }^{66-71}$

Ovarian-Adnexal Reporting and Data System (0-RADS)

The Ovarian-Adnexal Reporting and Data System (0-RADS) lexicon for ultrasound was published in 2018, providing a standardized glossary that includes all appropriate descriptors and definitions of the characteristic ultrasound appearance of normal ovaries and various adnexal lesions. ${ }^{72}{ }^{73}$ The 0-RADS ultrasound working group developed an adnexal mass triage system based either on the 0-RADS descriptors or on the risk of malignancy assigned to the mass using the IOTA ADNEX model to classify ovarian tumors into different risk categories. ${ }^{74}$ However, at present, neither the triage system nor the 0-RADS descriptors have been externally validated. Basha et $\mathrm{al}^{75}$ determined the malignancy rates, validity, and reliability of the 0-RADS approach when applied to a database of 647 adnexal masses collected before the development of the 0 -RADS system. In this retrospective study, the 0-RADS system had significantly higher sensitivity than did the GI-RADS system and the IOTA Simple Rules, with a non-significant slightly lower specificity compared with both GI-RADS and IOTA Simple Rules, and with similar reliability.

Statements on ultrasonography (Statements 1-6)

1. Subjective assessment by expert (Level III) ultrasound examiners has the best performance to distinguish between benign and malignant ovarian tumors.

- Level of evidence: 1a

- Grade of statement:A 
Table 2 Summary of main models and scoring systems for pre-operative diagnosis of ovarian tumors (modified from reference 63)

\begin{tabular}{ll}
\hline Model or system: type & Predictor variables \\
\hline Simple descriptors: & Benign descriptor (BD) 1: unilocular tumor \\
classification as benign or & with ground-glass echogenicity in a pre- \\
malignant & menopausal woman \\
& BD2: unilocular tumor with mixed echogenicity \\
& and acoustic shadows in a pre-menopausal \\
& woman \\
& BD3: unilocular anechoic tumor with regular \\
& walls and maximum diameter of lesion $<10 \mathrm{~cm}$ \\
& BD4: remaining unilocular tumor with regular \\
& walls \\
& Malignant descriptor (MD) 1: Tumor with \\
ascites and at least moderate color Doppler & blood flow in a post-menopausal woman \\
& MD2: age $>50$ years and CA $125>100 \mathrm{U} / \mathrm{mL}$
\end{tabular}

RMI:

score

Simple Rules: classification as benign, inconclusive or malignant

LR2: risk model based on logistic regression

Simple Rules risk: risk model based on logistic regression

\section{ADNEX without CA} 125: risk model based on multinomial logistic regression

\section{Remarks}

No risk estimates

Based on clinical, ultrasound and CA 125 information

Possible to calculate result without computer

CA 125, menopausal status, ultrasound score based on five binary ultrasound variables (multilocular cyst, solid areas, bilateral lesions, ascites, evidence of metastases on abdominal ultrasound)

No risk estimates

Based on clinical, ultrasound and CA 125

information

Possible to calculate result without computer Online calculators available

Classification based on 10 binary features - five No risk estimates

benign and five malignant features:

Benign features: unilocular cyst, smooth multilocular cyst with largest diameter $<100$ $\mathrm{mm}$, presence of solid areas with largest diameter $<7 \mathrm{~mm}$, acoustic shadows, no vascularization on color Doppler Malignant features: irregular solid tumor, irregular multilocular solid tumor with largest diameter $\geq 100 \mathrm{~mm}$, presence of ascites, $\geq 4$ papillary projections, very strong vascularization on color Doppler

Age (years), presence of acoustic shadows, presence of ascites, presence of papillary projections with blood flow, maximum diameter of largest solid component, irregular internal cyst walls

Classification into only three groups

Based on dichotomized ultrasound features

Easy to use without computer

Available as smartphone app

Risk estimates

Based on clinical and ultrasound information Requires computer

Available as smartphone app

The 10 binary features used in the Simple Rules, type of center (oncology center vs other)

Risk estimates

Based on dichotomized ultrasound features Developed to add risk estimates for Simple Rules Available as online calculator; available in ultrasound machines from some manufacturers

Age (years), maximum diameter of lesion $(\mathrm{mm})$, Risk estimates

maximum diameter of largest solid component Also estimates risk of four subtypes of $(\mathrm{mm})$, number of papillary projections (ordinal), malignancy presence of acoustic shadows, presence of Based on clinical and ultrasound information ascites, presence of more than 10 cyst locules, Subjective predictors are avoided a priori (eg, type of center (oncology center vs other) color score or irregular cyst walls)

Requires computer

Available as smartphone app and as online calculator; available in ultrasound machines from some manufacturers 
Table 2 Continued

\begin{tabular}{lll}
\hline Model or system: type & Predictor variables & Remarks \\
\hline $\begin{array}{ll}\text { ADNEX with CA 125: } \\
\text { risk model based on }\end{array}$ & Same variables as for ADNEX without CA 125, & Risk estimates \\
multinomial logistic & and additionally serum CA 125 (IU/L) & Also estimates risk of four subtypes of \\
regression & malignancy \\
& Based on clinical, ultrasound, and CA 125 \\
& information \\
& Subjective predictors are avoided a priori (eg, \\
& color score or irregular cyst walls) \\
& Requires computer \\
& Available as smartphone app and as online \\
& calculator; available in ultrasound machines from \\
& some manufacturers
\end{tabular}

ADNEX, Assessment of Different NEoplasias in the adneXa; CA 125, cancer antigen 125; RMI, risk of malignancy index.

- Consensus: yes, 95\% ( $n=19)$; no, $0 \%(n=0)$; abstain, $5 \%$ $(n=1)$

2. If an expert ultrasound examiner is not available, the use of ultrasound-based diagnostic models can assist clinicians to distinguish between benign and malignant ovarian tumors.

- Level of evidence: 2a

- Grade of statement: B

- Consensus: yes, $90 \%(n=18) ; n$, $0 \%(n=0)$; abstain, $10 \%$ $(n=2)$

3. Ultrasound-based diagnostic models (IOTA Simple Rules risk model or IOTA ADNEX model) are preferable to CA 125 level, HE4 level, or ROMA as they are superior in distinguishing between benign and malignant ovarian tumors.

- Level of evidence: $2 b$

- Grade of statement: B

- Consensus: yes, 95\% ( $n=19)$; no, $0 \%(n=0)$; abstain, $5 \%$ $(n=1)$

4. The IOTA ADNEX model and the IOTA Simple Rules risk model are recommended as they outperform existing morphological scoring systems, including the RMI.

- Level of evidence: $1 b$

- Grade of statement: A

- Consensus: yes, $95 \%(n=19) ;$ no, $0 \%(n=0)$; abstain, $5 \%$ $(n=1)$

5. The IOTA ADNEX model is a multiclass model and is helpful to differentiate between benign tumors, borderline tumors, earlyor advanced-stage ovarian cancer, and secondary metastatic tumors.

- Level of evidence: 3b

- Grade of statement: C

- Consensus: yes, $85 \%(n=17)$; no, $0 \%(n=0)$; abstain, $15 \%$ $(n=3)$

6. The threshold risk of there being a secondary metastatic tumor (as predicted by the IOTA ADNEX model), above which additional investigations to detect the primary organ of origin should be triggered, is $10 \%$.

- Level of evidence: 5

- Grade of statement: D

- Consensus: $5 \%$ threshold, $10 \%(n=2) ; 10 \%$ threshold, $75 \%$ $(n=15) ; 15 \%$ threshold, $0 \%(n=0) ; 20 \%$ threshold, $0 \%(n=0)$; abstain, $15 \%(n=3)$
Levels of evidence and grades are described in Online supplemental appendix 2 .

\section{Tumor markers}

According to a systematic quantitative review assessing the accuracy of CA 125 level in the diagnosis of benign, borderline, and malignant ovarian tumors, CA 125 is the best available single-protein biomarker identified to date. ${ }^{76}$ Although it lacks sensitivity and specificity for early stages of the disease and has a relatively low specificity overall, it can help direct treatment options in patients with suspicious ovarian masses. Pooled analyses have highlighted that a high body mass index and ethnicity might influence CA 125 levels, representing an additional diagnostic challenge. ${ }^{77}$ Other factors that influence CA 125 levels are the age of the patient, pregnancy, inflammatory processes, and the presence of fibroids or endometriosis. ${ }^{77-80}$

Multiple studies, including meta-analyses, have highlighted the role of HE4 as a potential complement to CA 125, especially in differentiating benign endometriotic and inflammatory lesions in younger women. ${ }^{251-103}$ Additional tumor markers (as in the ROMA test) have failed to improve significantly the discrimination between benign and malignant masses compared with CA 125 alone. ${ }^{53} 818491$ 96-109 The combination of a more extended tumor marker profile, including the addition of carcinoembryonic antigen (CEA) and/or carbohydrate antigen (CA 19-9) to CA 125, is useful mainly for differentiating between metastatic tumors from the gastrointestinal tract or pancreas and primary ovarian malignancy. ${ }^{110-113}$

Statements on tumor markers (Statements 7-12)

7. CA 125 is the best single-protein biomarker for the preoperative characterization of ovarian tumors. However, it is not useful as a screening test for ovarian cancer.

- Level of evidence: $2 b$

- Grade of statement: B

- Consensus: yes, 95\% ( $n=19)$; no, $0 \%(n=0)$; abstain, $5 \%$ $(n=1)$

8. Neither HE4 nor ROMA improves the discrimination between benign and malignant masses compared with CA 125 alone.

- Level of evidence: $2 b$

- Grade of statement: B

- Consensus: yes, $70 \%(n=14) ; n 0,0 \%(n=0)$; abstain, 30\% $(n=6)$ 


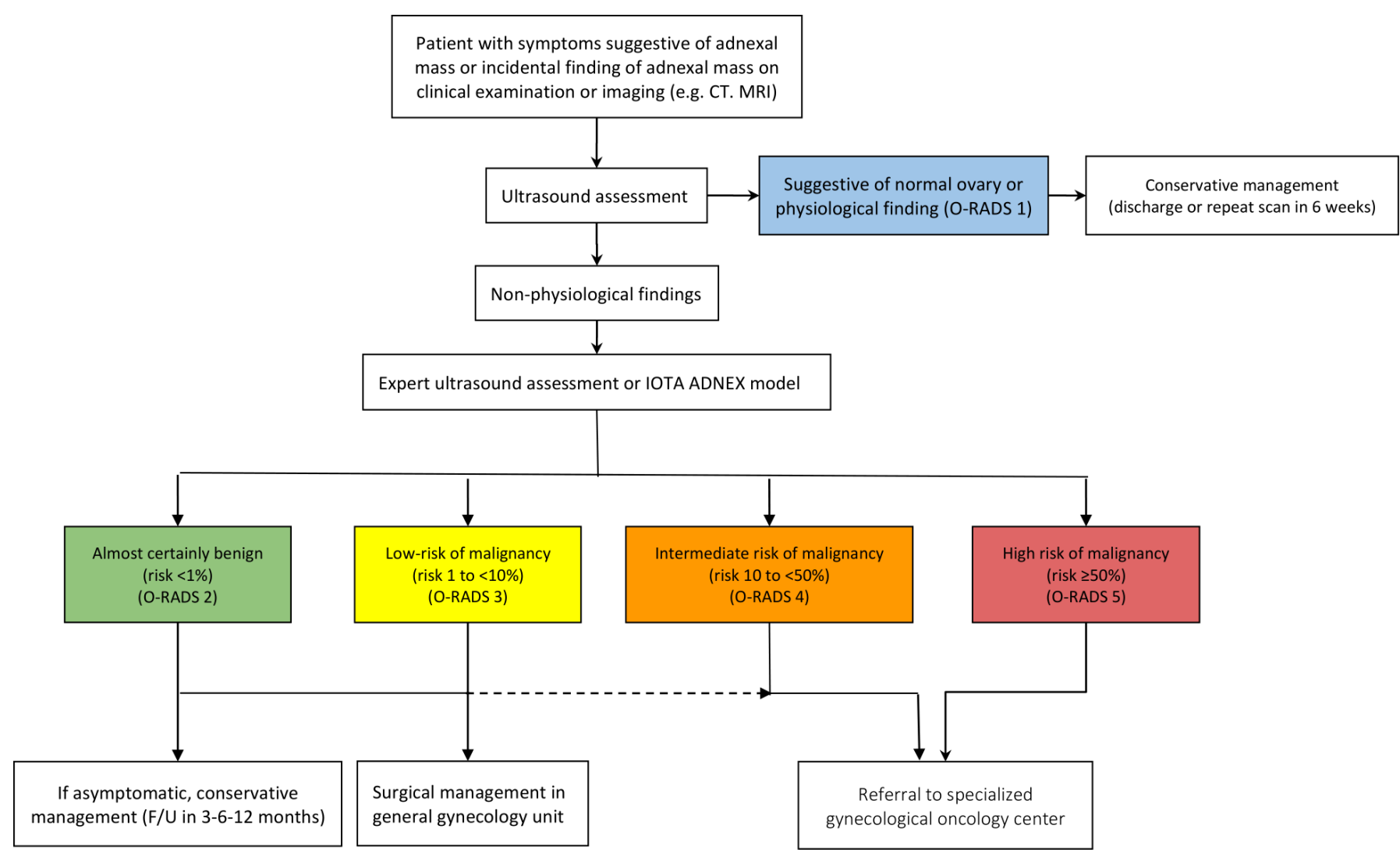

Figure 2 Flowchart of steps recommended to distinguish between benign and malignant tumors and to direct patients towards appropriate treatment pathway. CT, computed tomography; F/U, follow-up; IOTA ADNEX, International Ovarian Tumour Analysis Group Assessment of Different NEoplasias in the adneXa; MRI, magnetic resonance imaging; O-RADS, OvarianAdnexal Reporting and data system.

9. CA 125 does not increase the performance of ultrasoundbased risk models to distinguish between benign and malignant tumors.

- Level of evidence: $2 b$

- Grade of statement: B

- Consensus: yes, $60 \%$ ( $n=12)$; no, 10\% ( $n=2)$; abstain, 30\% $(\mathrm{n}=6)$

10. CA 125 is helpful as a biomarker in cases of suspected malignancy and it helps to distinguish between sub-types of malignant tumors, such as borderline and early- and advancedstage primary ovarian cancers and secondary metastatic tumors.

- Level of evidence: $2 b$

- Grade of statement: B

- Consensus: yes, 90\% ( $n=18)$; no, $5 \%(n=1)$; abstain, $5 \%$ $(n=1)$

11. CEA may be useful in specific cases to differentiate between primary ovarian cancer and secondary (ovarian) tumors.

- Level of evidence: $3 b$

- Grade of statement: C

- Consensus: yes, $90 \%(n=18) ; n o, 0 \%(n=0)$; abstain, $10 \%$ $(\mathrm{n}=2)$

12. CA 19-9 can help to differentiate secondary metastatic tumors in the ovary.

- Level of evidence: $3 b$

- Grade of statement: C
- Consensus: yes, $75 \%(n=15) ; n n, 5 \%(n=1)$; abstain, $20 \%$ $(\mathrm{n}=4)$

Levels of evidence and grades are described in Online supplemental appendix 2.

\section{Magnetic resonance imaging/computed tomography/positron emission tomography-computed tomography}

Magnetic resonance imaging

Several reports have found that magnetic resonance imaging (MRI), alone or in combination with computed tomography (CT), predicts accurately the presence of peritoneal carcinomatosis in patients undergoing pre-operative evaluation for cytoreductive surgery, particularly when the assessment is carried out by an experienced radiologist. ${ }^{114-117}$ Recently, a prospective study reported higher specificity of the IOTA LR2 model compared with subjective interpretation of MRI findings by an experienced radiologist, as well as similar sensitivities for both imaging modalities for discriminating between benign and malignant tumors. ${ }^{118}$ The addition of diffusion-weighted techniques to conventional imaging modalities has been shown in multiple pooled studies to increase diagnostic accuracy in discriminating between benign tumors and ovarian cancer, especially in the Caucasian population, with data even suggesting a value in predicting resectability. ${ }^{119-123}$ However, the true extent of such a benefit needs to be validated further in multicenter large-scale prospective randomized studies, which are currently being designed or underway. ${ }^{121}$ The addition of 


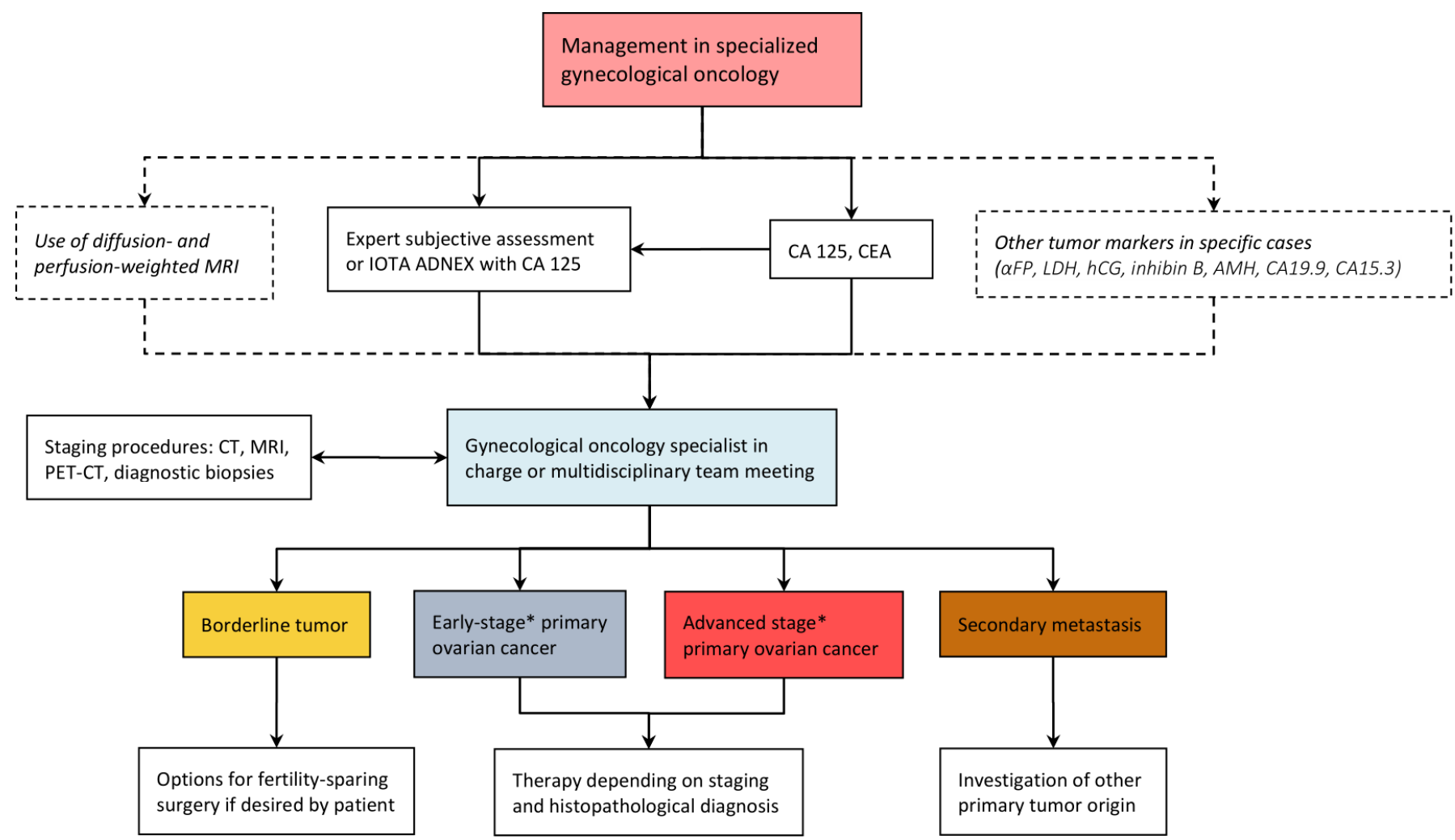

Figure 3 Flowchart of steps necessary to differentiate between subgroups of malignancy and extent of disease within gynecological oncology centers. *Early stage and advanced stage might differ according to different ADNEX models (stage I vs stages III-IV) and oncologically (stages I-II vs stages I-IV). $\alpha$ FP, alpha-fetoprotein; AMH, anti-Müllerian hormone; CA 125, cancer antigen 125; CA 15-3, cancer antigen 15-3; CA 19-9, carbohydrate antigen 19-9; CEA, carcinoembryonic antigen; CT, computed tomography; hCG, human chorionic gonadoptropin; IOTA ADNEX, International Ovarian Tumor Analysis group Assessment of Different NEoplasias in the adneXa; LDH, lactate dehydrogenase; MRI, magnetic resonance imaging; PET-CT, positron emission tomography-computed tomography.

quantitative dynamic contrast-enhanced MRI to diffusion-weighted imaging and anatomical MRI sequences and the development of a 5-point scoring system (0-RADS MRI score) is another modern diagnostic development with promising potential for the differentiation between benign and malignant adnexal masses in cases in which ultrasound is unable to arrive at a clear diagnosis (ie, indeterminate masses). When this technique is enhanced with volume quantification, it can help to discriminate between type I and type II epithelial ovarian cancers. ${ }^{124-130}$ However, there are only limited data available on the impact of these modern MRI techniques on clinical decision-making, and further studies are needed with larger sample populations. ${ }^{131}$

\section{Computed tomography}

Dedicated multidetector CT protocols with standardized peritoneal carcinomatosis index forms are the most common diagnostic tool used in routine clinical practice to assess the extent of tumor dissemination and the presence of peritoneal carcinomatosis. ${ }^{132-136} \mathrm{~A}$ radiological peritoneal carcinomatosis index applied at pre-operative CT within an expert setting has been shown to have low performance scores as a triage test to identify patients who are likely to have complete cytoreduction to no macroscopic residual disease. ${ }^{137}$ On retrospective analysis, pre-operative CT imaging showed high specificity but rather low sensitivity in detecting tumor involvement at key sites in ovarian cancer surgery. ${ }^{136}$ Multiple studies that have attempted to cross-validate the accuracy of CT scans in predicting unresectable disease and incomplete cytoreduction have shown a substantial drop in accuracy rates when attempts have been made to validate them in other cohorts. ${ }^{138-145}$ Thus, CT should not be used as the sole tool to predict the resectability of peritoneal carcinomatosis and exclude patients from surgery; rather, the full clinical context should be taken into account. Its widespread availability makes CT useful as a first-line diagnostic tool to identify patients who should not be selected for cytoreductive surgery, such as those with large/multifocal intra-parenchymatous distant metastases, acute thromboembolic events, or secondary metastatic tumors that limit the prognosis. The role of radiomics as an additional quantitative mathematical segmentation of conventional pre-operative CT images has shown some promising results in preliminary studies; however, larger studies are necessary for validation before this technique is implemented in clinical practice. ${ }^{146}$

Positron emission tomography-computed tomography Positron emission tomography-computed tomography (PET-CT) may be useful in differentiating malignant from borderline or benign ovarian tumors, with the limitation that its diagnostic performance can be impacted negatively by certain tumor histological sub-types due to the lower fluorodeoxyglucose uptake in clear cell and mucinous invasive subtypes. ${ }^{147-152}$ PET-CT can also play a role as an additional technique in the diagnosis of 
lymph node metastases, especially outside the abdominal cavity, or in characterizing unclear lesions in key areas that would alter clinical management (eg, chest lesions). ${ }^{153-155}$ However, PET-CT does not seem to be a relevant additional diagnostic modality for the true extent of peritoneal spread of ovarian cancer, specifically bowel and mesenteric serosa, and therefore fails to predict resectability in those key sites, especially in the presence of low-volume disease. ${ }^{156}$ Furthermore, PET-CT has been shown to have a low diagnostic value in differentiating borderline from benign tumors and should therefore not be used in clinical decision-making processes in that context, especially when considering fertility-sparing procedures. ${ }^{147} 148152$

\section{Statements on MRI, CT, and PET/CT (Statements 13-17)}

13. MRI with the inclusion of the functional sequences, dynamic contrast-enhanced and diffusion-weighted MRI, is not a firstline tool but may be used as a second-line tool after ultrasonography to further differentiate between benign, malignant, and borderline masses.

- Level of evidence: 2a

- Grade of statement: B

- Consensus: yes, $100 \%(n=20) ; n n, 0 \%(n=0)$; abstain, $0 \%$ $(n=0)$

14. PET-CT and whole-body diffusion MRI as a second step can help to detect non-ovarian origin of secondary metastatic tumors if suspicions are raised by the initial ultrasound examination.

- Level of evidence: 4

- Grade of statement: C

- Consensus: yes, $90 \%(n=18)$; no, $0 \%(n=0)$; abstain, $10 \%$ $(n=2)$

15. PET-CT cannot differentiate reliably between borderline and benign tumors.

- Level of evidence: 4

- Grade of statement: C

- Consensus: yes, 95\% ( $n=19)$; no, $0 \%(n=0)$; abstain, $5 \%$ $(n=1)$

16. Imaging alone cannot detect reliably the entire extent of either peritoneal carcinomatosis (especially in cases of small-volume carcinomatosis) or mesenteric and bowel serosal involvement.

- Level of evidence: $3 b$

- Grade of statement: B

- Consensus: yes, $85 \%(n=17)$; no, $5 \%(n=1)$; abstain, $10 \%$ $(n=2)$

17. Imaging alone should not be used for surgical decision-making in terms of the prediction of peritoneal tumor resectability.

- Level of evidence: 3b

- Grade of statement: B

- Consensus: yes, $80 \%(n=16) ;$ no, $15 \%(n=3)$; abstain, $5 \%$ $(n=1)$

Levels of evidence and grades are described in Online supplemental appendix 2 .

\section{Circulating cell-free DNA and circulating tumor cells}

Circulating cell-free DNA and circulating tumor cells as noninvasive cancer biomarkers and in non-invasive biopsy (sometimes called 'liquid biopsy') have been investigated in multiple studies. ${ }^{157-170}$ DNA methylation patterns in cell-free DNA show potential to detect a proportion of ovarian cancers up to 2 years in advance of diagnosis. They may potentially guide personalized treatment, even though validation studies are lacking. The prospective use of novel collection vials, which stabilize blood cells and reduce background DNA contamination in serum/ plasma samples, will facilitate the clinical implementation of liquid biopsy analyses. ${ }^{160} \mathrm{~A}$ prospective evaluation of the potential of cell-free DNA for the diagnosis of primary ovarian cancer using chromosomal instability as a read-out suggested that this might be a promising method to increase the specificity of the pre-surgical prediction of malignancy in patients with adnexal masses. ${ }^{168}$ However, even though these circulating biomarkers play a key role in understanding metastasis and tumorigenesis and provide comprehensive insight into tumor evolution and dynamics during treatment and disease progression, they still have not been established as part of routine clinical practice. ${ }^{157-159}$

One meta-analysis suggested that quantitative analysis of cell-free DNA has unsatisfactory sensitivity but acceptable specificity for the diagnosis of ovarian cancer. ${ }^{170}$ In a more recent meta-analysis, cell-free DNA appeared to be slightly better than CA 125 and similar to HE4 with respect to its diagnostic ability to discriminate individuals with from those without ovarian cancer. ${ }^{163}$ Nevertheless, the diagnostic value of cellfree DNA in patients with ovarian cancer remains unclear and the data should be interpreted with caution. Further large-scale prospective studies are strongly recommended to validate the potential applicability of using circulating cell-free DNA, alone or in combination with conventional markers, as a diagnostic biomarker for ovarian cancer, and to explore potential factors that may influence the accuracy of ovarian cancer diagnosis. ${ }^{170}$

Statement on circulating cell-free DNA and tumor cells

(Statement 18)

18. Circulating cell-free DNA and circulating tumor cells should not yet be used in routine clinical practice to differentiate between benign and malignant ovarian masses.

- Level of evidence: 4

- Grade of statement: C

- Consensus: yes, $85 \%(n=17)$; no, $5 \%(n=1)$; abstain, $10 \%$ $(n=2)$

Levels of evidence and grades are described in Online supplemental appendix 2.

\section{OVERVIEW OF CONSENSUS}

The experts also reached a consensus on a flowchart describing steps recommended to distinguish between benign and malignant tumors (Figure 2) and to direct patients towards appropriate treatment pathways. Ultrasonography is recommended as a first step to stratify patients with symptoms suggestive of an adnexal mass, and in those with an incidental finding of an adnexal mass on imaging. If the scan rules out normal ovaries and physiological changes (ie, rules out 0-RADS 1), the IOTA ADNEX model could be applied as a next step in order to determine the risk of malignancy. Any ultrasonographic examination in the case of a suspected ovarian mass should be performed by an expert sonographer. The resulting classification of the lesion into one of the 0-RADS categories ${ }^{2-5}$ can 
further guide the management and selection of patients for referral to a dedicated gynecological oncology center.

A consensus was also reached on further steps necessary to differentiate between sub-groups of malignancy and extent of disease within gynecological oncology centers (Figure 3). Ultrasound assessment by an expert or application of the IOTA ADNEX model in combination with the tumor marker profile (CA 125 and CEA, complemented with other markers in specific cases) can often indicate the specific sub-type of malignancy. If available, diagnosis of the primary lesion can be confirmed with diffusion- and perfusionweighted MRI, especially in cases in which fertility-sparing surgery is considered. A CT scan of chest, abdomen, and pelvis is mandatory before planned surgery for presumed malignancy, in order to exclude secondary cancers, thromboembolic events, and multifocal intraparenchymal distant metastases that would preclude resectability. The final management and treatment journey of the patient should be determined within an expert multidisciplinary setting, taking into account both the diagnostic findings and the overall patient profile, including symptoms, patient preferences and prior surgical, medical and reproductive history, with the ultimate aim of defining an individualized approach for every patient.

\section{Author affiliations}

${ }^{1}$ Gynecology and Obstetrics, University Hospitals KU Leuven, Leuven, Belgium

${ }^{2}$ Development and Regeneration, KU Leuven, Leuven, Belgium

${ }^{3}$ Clinical Research Unit, Institut Bergonie, Bordeaux, France

${ }^{4}$ Metabolism Digestion and Reproduction, Queen Charlotte's \& Chelsea Hospital, Imperial College, London, UK

${ }^{5}$ Woman, Child and Public Health, Fondazione Policlinico Universitario A Gemelli IRCCS, Rome, Italy

${ }^{6}$ Gynaecology and Gynaecological Oncology, Evangelische Kliniken Essen-Mitte, Essen, Germany

${ }^{7}$ Gynaecology and Obstetrics, University Clinic of Navarra, Madrid, Spain

${ }^{8}$ Obstetrics and Gynaecology, First Faculty of Medicine, Charles University and General University Hospital in Prague, Prague, Czech Republic

${ }^{9}$ Obstetrics and Gynecology, Medical University of Innsbruck, Innsbruck, Austria

${ }^{10}$ Radiology, University Clinic of Navarra, Madrid, Spain

${ }^{11}$ European Network of Gynaecological Cancers Advocacy Groups (ENGAGe)

Executive Group, Prague, Czech Republic

${ }^{12} \mathrm{KIU}$ - Patient Organisation for Women with Gynaecological Cancer, Copenhagen, Denmark

${ }^{13}$ Clinical Physiology, Nuclear Medicine \& PET, Rigshospitalet, Copenhagen

University Hospital, Copenhagen, Denmark

${ }^{14}$ Gynecology and Obstetrics, Gynecologic Oncology Unit, Santa Chiara Hospital,

Trento, Italy

${ }^{15}$ Gynaecological Surgery, Institut Gustave Roussy, Villejuif, France

${ }^{16}$ Gynecologic Oncology, Fondazione Policlinico Universitario A Gemelli IRCCS,

Rome, Italy

${ }^{17}$ Obstetrics and Gynecologic Oncology, University Hospital, Strasbourg, France

${ }^{18}$ Obstetrics and Gynecology, Università Cattolica del Sacro Cuore, Rome, Italy

${ }^{19}$ Obstetrics and Gynaecology and Gynaecologic Oncology, University Hospital

Leuven, Leuven Cancer Institute, Leuven, Belgium

${ }^{20}$ Radiology, University Hospitals Leuven, Leuven, Belgium

${ }^{21}$ Division of Translational MRI, Department of Imaging and Pathology, KU Leuven, Leuven, Belgium

${ }^{22}$ Gynaecologic Oncology, Hammersmith Hospital, Imperial College, London, UK

\section{Presented at}

This paper is being published simultaneously and jointly, in International Journal of Gynecological Cancer, Ultrasound in Obstetrics \& Gynecology and Facts, Views and Vision in ObGyn, by the European Society of Gynecological Oncology (ESGO), the International Society of Ultrasound in Obstetrics and Gynecology (ISUOG), the International Ovarian Tumour Analysis (IOTA) group and the European Society for Gynaecological Endoscopy (ESGE).
Twitter Guillermo Gallardo Madueño @ggallar

Acknowledgements The authors thank ESGO, ISUOG, IOTA, and ESGE for their support. We wish also to express sincere gratitude to Maciej Malecki (University Hospital Leuven, Leuven, Belgium) for providing technical support during the conference call.

Contributors The development group (including all authors) is collectively responsible for the decision to submit for publication. CF (chair), DT (chair), and FP (methodologist) wrote the first draft of the manuscript. All other contributors have actively given personal input, reviewed the manuscript, and have given final approval before submission.

Funding All costs relating to the development process were covered from ESGO, ISUOG, IOTA and ESGE funds. There was no external funding of the development process or manuscript production.

Competing interests DT: senior investigator FWO (Fund for Scientific Research Flanders), and research sponsored by Roche Diagnostics. KU Leuven has consultancy agreements with GE Healthcare, Samsung Healthcare, GSK, and Canon. TB: research sponsored by Roche Diagnostics, Samsung Medison and Illumina, and grants for traveling from Samsung Medison. LC: advisory boards for AstraZeneca, GSK, Takeda, and Roche. DC: advisory boards for Genmab, AstraZeneca, Roche, and Sotio. NC: advisory boards for AstraZeneca, Seattle Genetics, Mersana and eTheRNA Immunotherapies NV, grants for traveling from Roche, Genmab and Amgen, and educational fees from MSD and Medscape Oncology. AdB: advisory boards for Roche, AstraZeneca, GSK/Tesaro, BIOCAD, Clovis, Genmab/Seattle Genetics, Pfizer, and Amgen, and grants for traveling from Roche and AstraZeneca. IV: consulting activities for Amgen, AstraZeneca, Clovis Oncology, Carrick Therapeutics, Debiopharm International SA, Deciphera Pharmaceuticals, Elevar Therapeutics, F Hoffmann-La Roche Ltd, Genmab, GSK, Immunogen Inc, Medical University of Vienna, Mersana, Millenium Pharmaceuticals, MSD, Novocure, Octimet Oncology NV, Oncoinvent AS, Pharmamar, Sotioa.s, Tesaro Inc, Verastem Oncology, and Zentalis, contracted research (via KU Leuven) from Oncoinvent AS and Genmab, grants (corporate sponsored research) from Amgen and Roche, and accommodations/travel expenses from Amgen, MSD, Tesaro, AstraZeneca, and Roche. CF: advisory boards for Roche, GSK, Tesaro, AZ/MSD, Clovis, Sequana and Ethicon, and grants for traveling from GSK and Roche. FP, CL, DF, WF, GG, BL, AL, LM, PM, DQ, ACT, WV and GS: no conflicts of interest.

Patient consent for publication Not required.

Provenance and peer review Not commissioned; internally peer reviewed.

Data availability statement All data relevant to the study are included in the article or uploaded as supplementary information.

Open access This is an open access article distributed in accordance with the Creative Commons Attribution Non Commercial (CC BY-NC 4.0) license, which permits others to distribute, remix, adapt, build upon this work non-commercially, and license their derivative works on different terms, provided the original work is properly cited, an indication of whether changes were made, and the use is noncommercial. See: http://creativecommons.org/licenses/by-nc/4.0/.

\section{ORCID iDs}

Dirk Timmerman http://orcid.org/0000-0002-3707-6645

Tom Bourne http://orcid.org/0000-0003-1421-6059

Chiara Landolfo http://orcid.org/0000-0001-9808-7957

Luis Chiva http://orcid.org/0000-0002-1908-3251

David Cibula http://orcid.org/0000-0001-6387-9356

Nicole Concin http://orcid.org/0000-0002-9795-2643

Daniela Fischerova http://orcid.org/0000-0002-7224-3218

Wouter Froyman http://orcid.org/0000-0002-1398-9124

Guillermo Gallardo Madueño http://orcid.org/0000-0002-7502-3544

Denis Querleu http://orcid.org/0000-0002-3984-4812

Antonia Carla Testa http://orcid.org/0000-0003-2217-8726

Vincent Vandecaveye http://orcid.org/0000-0002-0800-3279

Christina Fotopoulou http://orcid.org/0000-0001-6375-9645

\section{REFERENCES}

1 Querleu D, Planchamp F, Chiva L, et al. European Society of Gynaecological Oncology (ESGO) guidelines for ovarian cancer surgery. Int J Gynecol Cancer 2017;27:1534-42.

2 Woo YL, Kyrgiou M, Bryant A, et al. Centralisation of services for gynaecological cancer. Cochrane Database Syst Rev 2012;4. 
3 Engelen MJA, Kos HE, Willemse PHB, et al. Surgery by consultant gynecologic oncologists improves survival in patients with ovarian carcinoma. Cancer 2006;106:589-98.

4 Froyman W, Landolfo C, De Cock B, et al. Risk of complications in patients with conservatively managed ovarian tumours (IOTA5): a 2 -year interim analysis of a multicentre, prospective, cohort study. Lancet Oncol 2019;20:448-58.

5 du Bois A, Rochon J, Pfisterer J, et al. Variations in institutional infrastructure, physician specialization and experience, and outcome in ovarian cancer: a systematic review. Gynecol Oncol 2009;112:422-36.

6 Elit LM, Bondy SJ, Paszat LP, et al. Surgical outcomes in women with ovarian cancer. Can J Surg 2008;51:346-54.

7 Vernooij F, Heintz P, Witteveen E, et al. The outcomes of ovarian cancer treatment are better when provided by gynecologic oncologists and in specialized hospitals: a systematic review. Gynecol Oncol 2007;105:801-12.

8 Kaijser J, Vandecaveye V, Deroose CM, et al. Imaging techniques for the pre-surgical diagnosis of adnexal tumours. Best Pract Res Clin Obstet Gynaecol 2014;28:683-95.

9 Valentin L, Hagen B, Tingulstad S, et al. Comparison of 'pattern recognition' and logistic regression models for discrimination between benign and malignant pelvic masses: a prospective cross validation. Ultrasound Obstet Gynecol 2001;18:357-65.

10 Timmerman $\mathrm{D}$. The use of mathematical models to evaluate pelvic masses; can they beat an expert operator? Best Pract Res Clin Obstet Gynaecol 2004;18:91-104.

11 Meys EMJ, Kaijser J, Kruitwagen RFPM, et al. Subjective assessment versus ultrasound models to diagnose ovarian cancer: a systematic review and meta-analysis. Eur J Cancer 2016;58:17-29.

12 Valentin L. Prospective cross-validation of Doppler ultrasound examination and gray-scale ultrasound imaging for discrimination of benign and malignant pelvic masses. Ultrasound Obstet Gynecol 1999;14:273-83.

13 Timmerman D, Schwärzler P, Collins WP, et al. Subjective assessment of adnexal masses with the use of ultrasonography: an analysis of interobserver variability and experience. Ultrasound Obstet Gynecol 1999;13:11-16.

14 Yazbek J, Raju SK, Ben-Nagi J, et al. Effect of quality of gynaecological ultrasonography on management of patients with suspected ovarian cancer: a randomised controlled trial. Lancet Oncol 2008;9:124-31.

15 Education and Practical Standards Committee, European Federation of Societies for Ultrasound in Medicine and Biology. Minimum training recommendations for the practice of medical ultrasound. Ultraschall Med 2006;27:79-105.

16 Jacobs I, Oram D, Fairbanks J, et al. A risk of malignancy index incorporating CA 125, ultrasound and menopausal status for the accurate preoperative diagnosis of ovarian cancer. Br J Obstet Gynaecol 1990;97:922-9.

17 Chacón E, Dasí J, Caballero C, et al. Risk of ovarian malignancy algorithm versus risk malignancy index-I for preoperative assessment of adnexal masses: a systematic review and metaanalysis. Gynecol Obstet Invest 2019;84:591-8.

18 Westwood M, Ramaekers B, Lang S, et al. Risk scores to guide referral decisions for people with suspected ovarian cancer in secondary care: a systematic review and cost-effectiveness analysis. Health Technol Assess 2018;22:1-264.

19 Khoiwal K, Bahadur A, Kumari R, et al. Assessment of diagnostic value of serum CA-125 and risk of malignancy index scoring in the evaluation of adnexal masses. J Midlife Health 2019;10:192-6.

20 Dochez V, Randet M, Renaudeau C. Efficacy of HE4, CA125, risk of malignancy index and risk of ovarian malignancy index to detect ovarian cancer in women with presumed benign ovarian tumours: a prospective, multicentre trial. J Clin Med 2019;8.

21 Al-Musalhi K, Al-Kindi M, Ramadhan F, et al. Validity of cancer antigen-125 (CA-125) and risk of malignancy index (RMI) in the diagnosis of ovarian cancer. Oman Med J 2015;30:428-34.

22 Zhang S, Yu S, Hou W, et al. Diagnostic extended usefulness of RMI: comparison of four risk of malignancy index in preoperative differentiation of borderline ovarian tumors and benign ovarian tumors. J Ovarian Res 2019;12:87.

23 Bouzari Z, Yazdani S, Shirkhani Kelagar Z, et al. Risk of malignancy index as an evaluation of preoperative pelvic mass. Caspian $J$ Intern Med 2011;2:331-5.

24 Chopra S, Vaishya R, Kaur J. An evaluation of the applicability of the risk of malignancy index for adnexal masses to patients seen at a tertiary hospital in Chandigarh, India. J Obstet Gynaecol India 2015;65:405-10.
25 Al Musalhi K, Al Kindi M, Al Aisary F, et al. Evaluation of HE4, $\mathrm{CA}-125$, risk of ovarian malignancy algorithm (ROMA) and risk of malignancy index (RMI) in the preoperative assessment of patients with adnexal mass. Oman Med J 2016;31:336-44.

26 Javdekar R, Maitra N. Risk of malignancy index (RMI) in evaluation of adnexal mass. J Obstet Gynaecol India 2015;65:117-21.

27 Aktürk E, Karaca RE, Alanbay I, et al. Comparison of four malignancy risk indices in the detection of malignant ovarian masses. J Gynecol Oncol 2011;22:177-82.

28 Hada A, Han L-P, Chen Y, et al. Comparison of the predictive performance of risk of malignancy indexes 1-4, HE4 and risk of malignancy algorithm in the triage of adnexal masses. J Ovarian Res 2020;13:46.

29 Anton C, Carvalho FM, Oliveira El, et al. A comparison of CA125, HE4, risk ovarian malignancy algorithm (ROMA), and risk malignancy index (RMI) for the classification of ovarian masses. Clinics 2012;67:437-41.

30 Moore RG, Brown AK, Miller MC, et al. The use of multiple novel tumor biomarkers for the detection of ovarian carcinoma in patients with a pelvic mass. Gynecol Oncol 2008;108:402-8.

31 Kaijser J, Van Gorp T, Sayasneh A, et al. Differentiating stage I epithelial ovarian cancer from benign disease in women with adnexal tumors using biomarkers or the ROMA algorithm. Gynecol Oncol 2013;130:398-9.

32 Timmerman D, Van Calster B, Jurkovic D, et al. Inclusion of CA-125 does not improve mathematical models developed to distinguish between benign and malignant adnexal tumors. J Clin Oncol 2007;25:4194-200.

33 Timmerman D, Valentin L, Bourne TH, et al. Terms, definitions and measurements to describe the sonographic features of adnexal tumors: a consensus opinion from the International Ovarian Tumor Analysis (IOTA) group. Ultrasound Obstet Gynecol 2000;16:500-5.

34 Timmerman D, Testa AC, Bourne T, et al. Logistic regression model to distinguish between the benign and malignant adnexal mass before surgery: a multicenter study by the International Ovarian Tumor Analysis group. J Clin Oncol 2005;23:8794-801.

35 Timmerman D, Van Calster B, Testa A, et al. Predicting the risk of malignancy in adnexal masses based on the simple rules from the International Ovarian Tumor Analysis group. Am J Obstet Gynecol 2016;214:424-37.

36 Van Calster B, Van Hoorde K, Valentin L, et al. Evaluating the risk of ovarian cancer before surgery using the ADNEX model to differentiate between benign, borderline, early and advanced stage invasive, and secondary metastatic tumours: prospective multicentre diagnostic study. BMJ 2014;349:g5920.

37 Van Holsbeke C, Van Calster B, Bourne T, et al. External validation of diagnostic models to estimate the risk of malignancy in adnexal masses. Clin Cancer Res 2012;18:815-25.

38 Sayasneh A, Wynants L, Preisler J, et al. Multicentre external validation of IOTA prediction models and RMI by operators with varied training. Br J Cancer 2013;108:2448-54.

39 Timmerman D, Testa AC, Bourne T, et al. Simple ultrasoundbased rules for the diagnosis of ovarian cancer. Ultrasound Obstet Gynecol 2008;31:681-90.

40 Timmerman D, Ameye L, Fischerova D, et al. Simple ultrasound rules to distinguish between benign and malignant adnexal masses before surgery: prospective validation by IOTA group. BMJ 2010;341:c6839.

41 Hartman CA, Juliato CRT, Sarian LO, et al. Ultrasound criteria and CA 125 as predictive variables of ovarian cancer in women with adnexal tumors. Ultrasound Obstet Gynecol 2012;40:360-6.

42 Alcazar JL, Pascual MA, Olartecoechea B, et al. IOTA simple rules for discriminating between benign and malignant adnexal masses: prospective external validation. Ultrasound Obstet Gynecol 2013;42:467-71.

43 Tantipalakorn C, Wanapirak C, Khunamornpong S, et al. IOTA simple rules in differentiating between benign and malignant ovarian tumors. Asian Pac J Cancer Prev 2014;15:5123-6.

44 Nunes N, Ambler G, Foo X, et al. Use of IOTA simple rules for diagnosis of ovarian cancer: meta-analysis. Ultrasound Obstet Gynecol 2014;44:503-14.

45 Ruiz de Gauna B, Rodriguez D, Olartecoechea B, et al. Diagnostic performance of IOTA simple rules for adnexal masses classification: a comparison between two centers with different ovarian cancer prevalence. Eur J Obstet Gynecol Reprod Biol 2015;191:10-14.

46 Knafel A, Banas T, Nocun A, et al. The prospective external validation of international ovarian tumor analysis (IOTA) simple rules in the hands of level I and II examiners. Ultraschall Med 2016;37:516-23. 
47 Ameye L, Timmerman D, Valentin L, et al. Clinically oriented threestep strategy for assessment of adnexal pathology. Ultrasound Obstet Gynecol 2012;40:582-91.

48 Kaijser J, Sayasneh A, Van Hoorde K, et al. Presurgical diagnosis of adnexal tumours using mathematical models and scoring systems: a systematic review and meta-analysis. Hum Reprod Update 2014;20:449-62.

49 American College of Obstetricians and Gynecologists' Committee on Practice Bulletins-Gynecology. Practice Bulletin No. 174: evaluation and management of adnexal masses. Obstet Gynecol 2016;128:e210-26.

50 Royal College of Obstetricians and Gynaecologists. Management of suspected ovarian masses in premenopausal women, 2011. Green-top guideline no 62. Available: https://www.rcog.org.uk/ globalassets/documents/guidelines/gtg_62.pdf

51 Glanc P, Benacerraf B, Bourne T, et al. First international consensus report on adnexal masses: management recommendations. $J$ Ultrasound Med 2017;36:849-63.

52 Nunes N, Ambler G, Foo X, et al. Comparison of two protocols for the management of asymptomatic postmenopausal women with adnexal tumours - a randomised controlled trial of RMI/RCOG vs Simple Rules. Br J Cancer 2017;116:584-91.

53 Piovano E, Cavallero C, Fuso L, et al. Diagnostic accuracy and cost-effectiveness of different strategies to triage women with adnexal masses: a prospective study. Ultrasound Obstet Gynecol 2017;50:395-403.

54 Alcázar JL, Pascual MA, Graupera B, et al. External validation of IOTA simple descriptors and simple rules for classifying adnexal masses. Ultrasound Obstet Gynecol 2016;48:397-402.

55 Sayasneh A, Ferrara L, De Cock B, et al. Evaluating the risk of ovarian cancer before surgery using the ADNEX model: a multicentre external validation study. $\mathrm{Br} J$ Cancer 2016;115:542-8.

56 Araujo KG, Jales RM, Pereira PN, et al. Performance of the IOTA ADNEX model in preoperative discrimination of adnexal masses in a gynecological oncology center. Ultrasound Obstet Gynecol 2017;49:778-83.

57 Meys EMJ, Jeelof LS, Achten NMJ, et al. Estimating risk of malignancy in adnexal masses: external validation of the ADNEX model and comparison with other frequently used ultrasound methods. Ultrasound Obstet Gynecol 2017;49:784-92.

58 Szubert S, Wojtowicz A, Moszynski R, et al. External validation of the IOTA ADNEX model performed by two independent gynecologic centers. Gynecol Oncol 2016;142:490-5.

59 Van Calster B, Steyerberg EW, Bourne T, et al. Flawed external validation study of the ADNEX model to diagnose ovarian cancer. Gynecol Oncol Rep 2016;18:49-50.

60 Van Calster B. External validation of ADNEX model for diagnosing ovarian cancer: evaluating performance of differentiation between tumor subgroups. Ultrasound Obstet Gynecol 2017;50:406-7.

61 Wynants L, Timmerman D, Verbakel JY, et al. Clinical utility of risk models to refer patients with adnexal masses to specialized oncology care: multicenter external validation using decision curve analysis. Clin Cancer Res 2017;23:5082-90.

62 Viora E, Piovano E, Baima Poma C, et al. The ADNEX model to triage adnexal masses: an external validation study and comparison with the IOTA two-step strategy and subjective assessment by an experienced ultrasound operator. Eur J Obstet Gynecol Reprod Biol 2020;247:207-11.

63 Van Calster B, Valentin L, Froyman W, et al. Validation of models to diagnose ovarian cancer in patients managed surgically or conservatively: multicentre cohort study. BMJ 2020;370:m2614.

64 Amor F, Vaccaro H, Alcázar JL, et al. Gynecologic imaging reporting and data system: a new proposal for classifying adnexal masses on the basis of sonographic findings. J Ultrasound Med 2009;28:285-91.

65 Amor F, Alcázar JL, Vaccaro H, et al. Gl-RADS reporting system for ultrasound evaluation of adnexal masses in clinical practice: a prospective multicenter study. Ultrasound Obstet Gynecol 2011;38:450-5.

66 Koneczny J, Czekierdowski A, Florczak M, et al. The use of sonographic subjective tumor assessment, IOTA logistic regression model 1, IOTA Simple Rules and GI-RADS system in the preoperative prediction of malignancy in women with adnexal masses. Ginekol Pol 2017;88:647-53.

67 Zheng $\mathrm{H}$, Tie $\mathrm{Y}$, Wang $\mathrm{X}$, et al. Assessment of the diagnostic value of using serum CA125 and GI-RADS system in the evaluation of adnexal masses. Medicine 2019;98:e14577.

68 Zhang T, Li F, Liu J, et al. Diagnostic performance of the Gynecology Imaging Reporting and Data System for malignant adnexal masses. Int J Gynaecol Obstet 2017;137:325-31.
69 Behnamfar F, Adibi A, Khadra H, et al. Diagnostic accuracy of gynecology imaging reporting and data system in evaluation of adnexal lesions. J Res Med Sci 2019;24.

70 Migda M, Bartosz M, Migda MS, et al. Diagnostic value of the gynecology imaging reporting and data system (GI-RADS) with the ovarian malignancy marker CA-125 in preoperative adnexal tumor assessment. J Ovarian Res 2018;11:92.

71 Basha MAA, Refaat R, Ibrahim SA. GI-RADS: diagnostic performance and inter-reviewer agreement. Eur Radiol 2019;29:5981-90.

72 Andreotti RF, Timmerman D, Benacerraf BR, et al. Ovarian-adnexal reporting lexicon for ultrasound: a white paper of the ACR OvarianAdnexal Reporting and Data System Committee. J Am Coll Radiol 2018;15:1415-29.

73 Erratum. J Am Coll Radiol 2019;16:403-6.

74 Andreotti RF, Timmerman D, Strachowski LM, et al. O-RADS US risk stratification and management system: a consensus guideline from the ACR Ovarian-Adnexal Reporting and Data System Committee. Radiology 2020;294:168-85.

75 Basha MAA, Metwally MI, Gamil SA, et al. Comparison of O-RADS, GI-RADS, and IOTA Simple Rules regarding malignancy rate, validity, and reliability for diagnosis of adnexal masses. Eur Radiol 2021;31:674-84.

76 Medeiros LR, Rosa DD, da Rosa MI, et al. Accuracy of CA 125 in the diagnosis of ovarian tumors: a quantitative systematic review. Eur J Obstet Gynecol Reprod Biol 2009;142:99-105.

77 Babic A, Cramer DW, Kelemen LE, et al. Predictors of pretreatment CA125 at ovarian cancer diagnosis: a pooled analysis in the Ovarian Cancer Association Consortium. Cancer Causes Control 2017;28:459-68.

78 Johnson CC, Kessel B, Riley TL, et al. The epidemiology of CA-125 in women without evidence of ovarian cancer in the prostate, lung, colorectal and ovarian cancer (PLCO) screening trial. Gynecol Oncol 2008;110:383-9.

79 Pauler DK, Menon U, Mclntosh M, et al. Factors influencing serum CA125II levels in healthy postmenopausal women. Cancer Epidemiol Biomarkers Prev 2001;10:489-93.

80 Cramer DW, Vitonis AF, Welch WR, et al. Correlates of the preoperative level of CA125 at presentation of ovarian cancer. Gynecol Oncol 2010;119:462-8.

81 Lycke M, Kristjansdottir B, Sundfeldt K. A multicenter clinical trial validating the performance of $\mathrm{HE} 4, \mathrm{CA} 125$, risk of ovarian malignancy algorithm and risk of malignancy index. Gynecol Oncol 2018;151:159-65.

82 Lin J, Qin J, Sangvatanakul V. Human epididymis protein 4 for differential diagnosis between benign gynecologic disease and ovarian cancer: a systematic review and meta-analysis. Eur $J$ Obstet Gynecol Reprod Biol 2013;167:81-5.

83 Richards A, Herbst U, Manalang J, et al. HE4, CA125, the Risk of Malignancy Algorithm and the Risk of Malignancy Index and complex pelvic masses - a prospective comparison in the preoperative evaluation of pelvic masses in an Australian population. Aust N Z J Obstet Gynaecol 2015;55:493-7.

84 Romagnolo C, Leon AE, Fabricio ASC, et al. HE4, CA125 and risk of ovarian malignancy algorithm (ROMA) as diagnostic tools for ovarian cancer in patients with a pelvic mass: an Italian multicenter study. Gynecol Oncol 2016;141:303-11.

85 Li F, Tie R, Chang K, et al. Does risk for ovarian malignancy algorithm excel human epididymis protein 4 and CA125 in predicting epithelial ovarian cancer: a meta-analysis. BMC Cancer 2012;12:258.

86 Wang J, Gao J, Yao H, et al. Diagnostic accuracy of serum HE4, CA125 and ROMA in patients with ovarian cancer: a meta-analysis. Tumour Biol 2014;35:6127-38.

87 Van Gorp T, Veldman J, Van Calster B, et al. Subjective assessment by ultrasound is superior to the risk of malignancy index (RMI) or the risk of ovarian malignancy algorithm (ROMA) in discriminating benign from malignant adnexal masses. Eur $J$ Cancer 2012;48:1649-56.

88 Stiekema A, Lok CAR, Kenter GG, et al. A predictive model combining human epididymal protein 4 and radiologic features for the diagnosis of ovarian cancer. Gynecol Oncol 2014;132:573-7.

89 Yanaranop M, Anakrat V, Siricharoenthai S, et al. Is the risk of ovarian malignancy algorithm better than other tests for predicting ovarian malignancy in women with pelvic masses? Gynecol Obstet Invest 2017:82:47-53.

90 Jacob F, Meier M, Caduff R, et al. No benefit from combining HE4 and CA125 as ovarian tumor markers in a clinical setting. Gynecol Oncol 2011;121:487-91.

91 Melo Ângela, Veríssimo R, Farinha M, et al. Discriminative value of CA-125, HE4, risk of malignancy index II (RMI-II) and risk of 
malignancy algorithm (ROMA) in the differential diagnosis of pelvic masses: conclusions from a referral centre in Portugal. J Obstet Gynaecol 2018;38:1140-5.

92 Jia M-M, Deng J, Cheng X-L, et al. Diagnostic accuracy of urine HE4 in patients with ovarian cancer: a meta-analysis. Oncotarget 2017;8:9660-71.

93 Huang J, Chen J, Huang Q. Diagnostic value of HE4 in ovarian cancer: a meta-analysis. Eur J Obstet Gynecol Reprod Biol 2018;231:35-42

94 Cao H, You D, Lan Z, et al. Prognostic value of serum and tissue HE4 expression in ovarian cancer: a systematic review with metaanalysis of 90 studies. Expert Rev Mol Diagn 2018;18:371-83.

95 Yu S, Yang HJ, Xie SQ, et al. Diagnostic value of HE4 for ovarian cancer: a meta-analysis. Clin Chem Lab Med 2012;50:1439-46.

96 Sandri MT, Bottari F, Franchi D, et al. Comparison of HE4, CA125 and ROMA algorithm in women with a pelvic mass: correlation with pathological outcome. Gynecol Oncol 2013;128:233-8.

97 Kim B, Park Y, Kim B. Diagnostic performance of CA 125, HE4, and risk of ovarian malignancy algorithm for ovarian cancer. J Clin Lab Anal 2019;33:e22624.

98 Kotowicz B, Fuksiewicz M, Sobiczewski P, et al. Clinical value of human epididymis protein 4 and the risk of ovarian malignancy algorithm in differentiating borderline pelvic tumors from epithelial ovarian cancer in early stages. Eur J Obstet Gynecol Reprod Biol 2015;194:141-6.

99 Shin $\mathrm{KH}$, Kim HH, Kwon BS, et al. Clinical usefulness of cancer antigen (CA) 125, human epididymis 4, and CA72-4 levels and risk of ovarian malignancy algorithm values for diagnosing ovarian tumors in Korean patients with and without endometriosis. Ann Lab Med 2020;40:40-7.

100 Terlikowska KM, Dobrzycka B, Witkowska AM, et al. Preoperative HE4, CA125 and ROMA in the differential diagnosis of benign and malignant adnexal masses. J Ovarian Res 2016;9:43.

101 Xu Y, Zhong R, He J, et al. Modification of cut-off values for HE4, CA125 and the ROMA algorithm for early-stage epithelial ovarian cancer detection: results from 1021 cases in South China. Clin Biochem 2016;49:32-40.

102 Yanaranop M, Jantarateptewan N, Tiyayon J, et al. Significance of serum human epididymis protein 4 and cancer antigen 125 in distinguishing type I and type II epithelial ovarian cancers. Int J Gynecol Cancer 2018;28:1058-65.

103 Zhang P, Wang C, Cheng L, et al. Comparison of HE4, CA125, and ROMA diagnostic accuracy: a prospective and multicenter study for Chinese women with epithelial ovarian cancer. Medicine 2015;94:e2402.

104 Choi H-J, Lee Y-Y, Sohn I, et al. Comparison of CA 125 alone and risk of ovarian malignancy algorithm (ROMA) in patients with adnexal mass: a multicenter study. Curr Probl Cancer 2020;44:100508.

105 Kaijser J, Van Gorp T, Smet M-E, et al. Are serum HE4 or ROMA scores useful to experienced examiners for improving characterization of adnexal masses after transvaginal ultrasonography? Ultrasound Obstet Gynecol 2014;43:89-97.

106 Chen X, Zhou H, Chen R, et al. Development of a multimarker assay for differential diagnosis of benign and malignant pelvic masses. Clin Chim Acta 2015;440:57-63.

107 Cui R, Wang Y, Li Y, et al. Clinical value of ROMA index in diagnosis of ovarian cancer: meta-analysis. Cancer Manag Res 2019;11:2545-51.

108 Huy NVQ, Van Khoa V, Tam LM, et al. Standard and optimal cut-off values of serum CA-125, HE4 and ROMA in preoperative prediction of ovarian cancer in Vietnam. Gynecol Oncol Rep 2018;25:110-4.

109 Shen F, Lu S, Peng Y, et al. Performance of ROMA based on architect CA 125 II and HE4 values in Chinese women presenting with a pelvic mass: a multicenter prospective study. Clin Chim Acta 2017;471:119-25.

110 Sagi-Dain L, Lavie O, Auslander R, et al. CEA in evaluation of adnexal mass: retrospective cohort analysis and review of the literature. Int J Biol Markers 2015;30:e394-400.

111 Sagi-Dain L, Lavie O, Auslander R, et al. CA 19-9 in evaluation of adnexal mass: retrospective cohort analysis and review of the literature. Int J Biol Markers 2015;30:e333-40.

112 Kelly PJ, Archbold P, Price JH, et al. Serum CA19.9 levels are commonly elevated in primary ovarian mucinous tumours but cannot be used to predict the histological subtype. J Clin Pathol 2010;63:169-73.

113 Bozkurt M, Yumru AE, Aral I. Evaluation of the importance of the serum levels of CA-125, CA15-3, CA-19-9, carcinoembryonic antigen and alpha fetoprotein for distinguishing benign and malignant adnexal masses and contribution of different test combinations to diagnostic accuracy. Eur J Gynaecol Oncol 2013;34:540-4.

114 Dohan A, Hoeffel C, Soyer P, et al. Evaluation of the peritoneal carcinomatosis index with CT and MRI. Br J Surg 2017;104:1244-9.

115 Low RN, Barone RM, Lucero J. Comparison of MRI and CT for predicting the peritoneal cancer index $(\mathrm{PCl})$ preoperatively in patients being considered for cytoreductive surgical procedures. Ann Surg Oncol 2015;22:1708-15.

116 Torkzad MR, Casta N, Bergman A, et al. Comparison between $\mathrm{MRI}$ and CT in prediction of peritoneal carcinomatosis index $(\mathrm{PCl})$ in patients undergoing cytoreductive surgery in relation to the experience of the radiologist. J Surg Oncol 2015;111:746-51.

117 Gadelhak B, Tawfik AM, Saleh GA, et al. Extended abdominopelvic $M R I$ versus $C T$ at the time of adnexal mass characterization for assessing radiologic peritoneal cancer index $(\mathrm{PCl})$ prior to cytoreductive surgery. Abdom Radiol 2019;44:2254-61.

118 Shimada K, Matsumoto K, Mimura T, et al. Ultrasound-based logistic regression model LR2 versus magnetic resonance imaging for discriminating between benign and malignant adnexal masses: a prospective study. Int J Clin Oncol 2018;23:514-21.

119 Dai G, Liang K, Xiao Z, et al. A meta-analysis on the diagnostic value of diffusion-weighted imaging on ovarian cancer. J Buon 2019;24:2333-40.

120 Meng X-F, Zhu S-C, Sun S-J, et al. Diffusion weighted imaging for the differential diagnosis of benign vs. malignant ovarian neoplasms. Oncol Lett 2016;11:3795-802.

121 Michielsen K, Dresen R, Vanslembrouck R, et al. Diagnostic value of whole body diffusion-weighted MRI compared to computed tomography for pre-operative assessment of patients suspected for ovarian cancer. Eur J Cancer 2017;83:88-98.

122 Espada M, Garcia-Flores JR, Jimenez M, et al. Diffusion-weighted magnetic resonance imaging evaluation of intra-abdominal sites of implants to predict likelihood of suboptimal cytoreductive surgery in patients with ovarian carcinoma. Eur Radiol 2013;23:2636-42.

123 Rizzo S, De Piano F, Buscarino V, et al. Pre-operative evaluation of epithelial ovarian cancer patients: role of whole body diffusion weighted imaging MR and CT scans in the selection of patients suitable for primary debulking surgery. A single-centre study. Eur $J$ Radiol 2020;123:108786.

124 Gity M, Parviz S, Saligheh Rad H, et al. Differentiation of benign from malignant adnexal masses by dynamic contrast-enhanced MRI (DCE-MRI): quantitative and semi-quantitative analysis at 3-Tesla MRI. Asian Pac J Cancer Prev 2019;20:1073-9.

125 Malek M, Oghabian Z, Tabibian E, et al. Comparison of qualitative (time intensity curve analysis), semi-quantitative, and quantitative multi-phase 3T DCEMRI parameters as predictors of malignancy in adnexal. Asian Pac J Cancer Prev 2019;20:1603-11.

126 Thomassin-Naggara I, Balvay D, Aubert E, et al. Quantitative dynamic contrast-enhanced MR imaging analysis of complex adnexal masses: a preliminary study. Eur Radiol 2012;22:738-45.

127 Carter JS, Koopmeiners JS, Kuehn-Hajder JE, et al. Quantitative multiparametric MRI of ovarian cancer. J Magn Reson Imaging 2013;38:1501-9.

$128 \mathrm{He} \mathrm{M}$, Song Y, Li H, et al. Histogram analysis comparison of monoexponential, advanced diffusion-weighted imaging, and dynamic contrast-enhanced MRI for differentiating borderline from malignant epithelial ovarian tumors. J Magn Reson Imaging 2020;52:257-68.

129 Li H-M, Feng F, Qiang J-W, et al. Quantitative dynamic contrastenhanced MR imaging for differentiating benign, borderline, and malignant ovarian tumors. Abdom Radiol 2018;43:3132-41.

130 Thomassin-Naggara I, Poncelet E, Jalaguier-Coudray A, et al. Ovarian-Adnexal Reporting Data System Magnetic Resonance Imaging (O-RADS MRI) score for risk stratification of sonographically indeterminate adnexal masses. JAMA Netw Open 2020;3:e1919896.

131 Dirrichs T, Bauerschlag D, Maass N, et al. Impact of multiparametric $\mathrm{MRI}(\mathrm{mMRI})$ on the therapeutic management of adnexal masses detected with transvaginal ultrasound (TVUS): an interdisciplinary management approach. Acad Radiol 2020;S1076-6332:30652-8.

132 Ahmed SA, Abou-Taleb H, Yehia A, et al. The accuracy of multidetector computed tomography and laparoscopy in the prediction of peritoneal carcinomatosis index score in primary ovarian cancer. Acad Radiol 2019;26:1650-8.

133 Byrom J, Widjaja E, Redman CWE, et al. Can pre-operative computed tomography predict resectability of ovarian carcinoma at primary laparotomy? BJOG 2002;109:369-75.

134 Esquivel J, Chua TC, Stojadinovic A, et al. Accuracy and clinical relevance of computed tomography scan interpretation of peritoneal cancer index in colorectal cancer peritoneal 
carcinomatosis: a multi-institutional study. J Surg Oncol 2010;102:565-70.

135 Marin D, Catalano C, Baski M, et al. 64-Section multi-detector row $\mathrm{CT}$ in the preoperative diagnosis of peritoneal carcinomatosis: correlation with histopathological findings. Abdom Imaging 2010;35:694-700.

136 Nasser S, Lazaridis A, Evangelou M, et al. Correlation of preoperative CT findings with surgical and histological tumor dissemination patterns at cytoreduction for primary advanced and relapsed epithelial ovarian cancer: a retrospective evaluation. Gynecol Oncol 2016;143:264-9.

137 Avesani G, Arshad M, Lu H, et al. Radiological assessment of peritoneal cancer index on preoperative CT in ovarian cancer is related to surgical outcome and survival. Radiol Med 2020;125:770-6.

138 Shim S-H, Lee SJ, Kim S-O, et al. Nomogram for predicting incomplete cytoreduction in advanced ovarian cancer patients. Gynecol Oncol 2015;136:30-6.

139 Dowdy SC, Mullany SA, Brandt KR, et al. The utility of computed tomography scans in predicting suboptimal cytoreductive surgery in women with advanced ovarian carcinoma. Cancer 2004;101:346-52.

140 Nelson BE, Rosenfield AT, Schwartz PE. Preoperative abdominopelvic computed tomographic prediction of optimal cytoreduction in epithelial ovarian carcinoma. J Clin Oncol 1993;11:166-72.

141 Bristow RE, Duska LR, Lambrou NC, et al. A model for predicting surgical outcome in patients with advanced ovarian carcinoma using computed tomography. Cancer 2000;89:1532-40.

142 Gemer O, Gdalevich M, Ravid M, et al. A multicenter validation of computerized tomography models as predictors of non-optimal primary cytoreduction of advanced epithelial ovarian cancer. Eur J Surg Oncol 2009;35:1109-12.

143 Axtell AE, Lee MH, Bristow RE, et al. Multi-institutional reciprocal validation study of computed tomography predictors of suboptima primary cytoreduction in patients with advanced ovarian cancer. $J$ Clin Oncol 2007;25:384-9.

144 Rutten MJ, van de Vrie R, Bruining A, et al. Predicting surgical outcome in patients with International Federation of Gynecology and Obstetrics stage III or IV ovarian cancer using computed tomography: a systematic review of prediction models. Int J Gynecol Cancer 2015;25:407-15.

$145 \mathrm{Kim} \mathrm{H}-J$, Choi CH, Lee Y-Y, et al. Surgical outcome prediction in patients with advanced ovarian cancer using computed tomography scans and intraoperative findings. Taiwan J Obstet Gynecol 2014;53:343-7.

$146 \mathrm{Lu} \mathrm{H}$, Arshad M, Thornton A, et al. A mathematical-descriptor of tumor-mesoscopic-structure from computed-tomography images annotates prognostic- and molecular-phenotypes of epithelial ovarian cancer. Nat Commun 2019;10:764.

147 Tanizaki Y, Kobayashi A, Shiro M, et al. Diagnostic value of preoperative SUVmax on FDG-PET/CT for the detection of ovarian cancer. Int J Gynecol Cancer 2014;24:454-60.

148 Kitajima K, Suzuki K, Senda M, et al. FDG-PET/CT for diagnosis of primary ovarian cancer. Nucl Med Commun 2011;32:549-53.

149 Nam EJ, Yun MJ, Oh YT, et al. Diagnosis and staging of primary ovarian cancer: correlation between PET/CT, Doppler US, and CT or MRI. Gynecol Oncol 2010;116:389-94.

150 Castellucci P, Perrone AM, Picchio M, et al. Diagnostic accuracy of 18F-FDG PET/CT in characterizing ovarian lesions and staging ovarian cancer: correlation with transvaginal ultrasonography, computed tomography, and histology. Nucl Med Commun 2007;28:589-95.

151 Risum S, Høgdall C, Loft A, et al. The diagnostic value of PET/CT for primary ovarian cancer--a prospective study. Gynecol Oncol 2007;105:145-9.

152 Yamamoto Y, Oguri H, Yamada R, et al. Preoperative evaluation of pelvic masses with combined 18F-fluorodeoxyglucose positron emission tomography and computed tomography. Int J Gynaecol Obstet 2008;102:124-7.

153 Dauwen H, Van Calster B, Deroose CM, et al. PET/CT in the staging of patients with a pelvic mass suspicious for ovarian cancer. Gynecol Oncol 2013;131:694-700.

154 Laghi A, Bellini D, Rengo M, et al. Diagnostic performance of computed tomography and magnetic resonance imaging for detecting peritoneal metastases: systematic review and metaanalysis. Radiol Med 2017;122:1-15.

155 Kim S-J, Lee S-W. Diagnostic accuracy of ${ }^{18}$ F-FDG PET/CT for detection of peritoneal carcinomatosis; a systematic review and meta-analysis. Br J Radiol 2018;91:20170519.
156 Michielsen K, Vergote I, Op de Beeck K, et al. Whole-body $\mathrm{MRI}$ with diffusion-weighted sequence for staging of patients with suspected ovarian cancer: a clinical feasibility study in comparison to CT and FDG-PET/CT. Eur Radiol 2014;24:889-901.

157 Barbosa A, Peixoto A, Pinto P, et al. Potential clinical applications of circulating cell-free DNA in ovarian cancer patients. Expert Rev Mol Med 2018;20:e6.

158 Chen Q, Zhang Z-H, Wang S, et al. Circulating cell-free DNA or circulating tumor DNA in the management of ovarian and endometrial cancer. Onco Targets Ther 2019;12:11517-30.

159 Giannopoulou L, Kasimir-Bauer S, Lianidou ES. Liquid biopsy in ovarian cancer: recent advances on circulating tumor cells and circulating tumor DNA. Clin Chem Lab Med 2018;56:186-97.

160 Widschwendter M, Zikan M, Wahl B, et al. The potential of circulating tumor DNA methylation analysis for the early detection and management of ovarian cancer. Genome Med 2017;9:116.

161 Guo Y-X, Neoh KH, Chang X-H, et al. Diagnostic value of HE4+ circulating tumor cells in patients with suspicious ovarian cancer. Oncotarget 2018;9:7522-33.

162 Kolostova K, Matkowski R, Jędryka M, et al. The added value of circulating tumor cells examination in ovarian cancer staging. Am J Cancer Res 2015;5:3363-75.

163 Li B, Pu K, Ge L, et al. Diagnostic significance assessment of the circulating cell-free DNA in ovarian cancer: an updated metaanalysis. Gene 2019;714:143993.

164 Li N, Zuo H, Chen L, et al. Circulating tumor cell detection in epithelial ovarian cancer using dual-component antibodies targeting EpCAM and FRo. Cancer Manag Res 2019;11:10939-48.

165 Lou E, Vogel RI, Teoh D, et al. Assessment of circulating tumor cells as a predictive biomarker of histology in women with suspected ovarian cancer. Lab Med 2018;49:134-9.

166 Phallen J, Sausen M, Adleff V, et al. Direct detection of earlystage cancers using circulating tumor DNA. Sci Trans/ Med 2017;9:eaan2415.

167 Suh DH, Kim M, Choi JY, et al. Circulating tumor cells in the differential diagnosis of adnexal masses. Oncotarget 2017;8:77195-206.

168 Vanderstichele A, Busschaert P, Smeets D, et al. Chromosomal instability in cell-free DNA as a highly specific biomarker for detection of ovarian cancer in women with adnexal masses. Clin Cancer Res 2017;23:2223-31.

169 Yu Z, Qin S, Wang H. Alter circulating cell-free DNA variables in plasma of ovarian cancer patients. J Obstet Gynaecol Res 2019;45:2237-42.

170 Zhou Q, Li W, Leng B, et al. Circulating cell free DNA as the diagnostic marker for ovarian cancer: a systematic review and meta-analysis. PLoS One 2016;11:e0155495.

171 Van Holsbeke C, Van Calster B, Guerriero S, et al. Endometriomas: their ultrasound characteristics. Ultrasound Obstet Gynecol 2010;35:730-40.

172 Paladini D, Testa A, Van Holsbeke C, et al. Imaging in gynecological disease (5): clinical and ultrasound characteristics in fibroma and fibrothecoma of the ovary. Ultrasound Obstet Gynecol 2009;34:188-95.

173 Demidov VN, Lipatenkova J, Vikhareva O, et al. Imaging of gynecological disease (2): clinical and ultrasound characteristics of Sertoli cell tumors, Sertoli-Leydig cell tumors and Leydig cell tumors. Ultrasound Obstet Gynecol 2008;31:85-91.

174 Savelli L, Testa AC, Timmerman D, et al. Imaging of gynecological disease (4): clinical and ultrasound characteristics of struma ovarii. Ultrasound Obstet Gynecol 2008;32:210-9.

175 Virgilio BA, De Blasis I, Sladkevicius P, et al. Imaging in gynecological disease (16): clinical and ultrasound characteristics of serous cystadenofibromas in adnexa. Ultrasound Obstet Gynecol 2019;54:823-30.

176 Moro F, Zannoni GF, Arciuolo D, et al. Imaging in gynecological disease (11): clinical and ultrasound features of mucinous ovarian tumors. Ultrasound Obstet Gynecol 2017;50:261-70.

177 Dierickx I, Valentin L, Van Holsbeke C, et al. Imaging in gynecological disease (7): clinical and ultrasound features of Brenner tumors of the ovary. Ultrasound Obstet Gynecol 2012;40:706-13.

178 Timor-Tritsch IE, Lerner JP, Monteagudo A, et al. Transvaginal sonographic markers of tubal inflammatory disease. Ultrasound Obstet Gynecol 1998;12:56-66.

179 Kurman RJ, Carcangiu ML, Herrington S. WHO classification of tumours of female reproductive organs. 4th edn. Lyon: IARC, 2014.

180 Franchi D, Boveri S, Fruscio R, et al. Imaging in gynecological disease (8): ultrasound characteristics of recurrent borderline ovarian tumors. Ultrasound Obstet Gynecol 2013;41:452-8. 


\section{Joint statement}

181 Moro F, Baima Poma C, Zannoni GF, et al. Imaging in gynecological disease (12): clinical and ultrasound features of invasive and non-invasive malignant serous ovarian tumors. Ultrasound Obstet Gynecol 2017:50:788-99.

182 Landolfo C, Valentin L, Franchi D, et al. Differences in ultrasound features of papillations in unilocular-solid adnexal cysts: a retrospective international multicenter study. Ultrasound Obstet Gynecol 2018;52:269-78.

183 Moro F, Magoga G, Pasciuto T, et al. Imaging in gynecological disease (13): clinical and ultrasound characteristics of endometrioid ovarian cancer. Ultrasound Obstet Gynecol 2018;52:535-43.

184 Pozzati F, Moro F, Pasciuto T, et al. Imaging in gynecological disease (14): clinical and ultrasound characteristics of ovarian clear cell carcinoma. Ultrasound Obstet Gynecol 2018;52:792-800.

185 Van Holsbeke C, Domali E, Holland TK, et al. Imaging of gynecological disease (3): clinical and ultrasound characteristics of granulosa cell tumors of the ovary. Ultrasound Obstet Gynecol 2008;31:450-6.

186 Guerriero S, Testa AC, Timmerman D, et al. Imaging of gynecological disease (6): clinical and ultrasound characteristics of ovarian dysgerminoma. Ultrasound Obstet Gynecol 2011;37:596-602

187 Anfelter P, Testa A, Chiappa V, et al. Imaging in gynecological disease (17): ultrasound features of malignant ovarian yolk sac tumors (endodermal sinus tumors). Ultrasound Obstet Gynecol 2020;56:276-84.

188 Corrigendum. Ultrasound Obstet Gynecol 2020;56.

189 Moro F, Castellano LM, Franchi D, et al. Imaging in gynecological disease: clinical and ultrasound characteristics of ovarian embryonal carcinomas, non-gestational choriocarcinomas and malignant mixed germ cell tumors. Ultrasound Obstet Gynecol 2020. doi:10.1002/uog.23529. [Epub ahead of print: 03 Nov 2020].

190 Testa AC, Ferrandina G, Timmerman D, et al. Imaging in gynecological disease (1): ultrasound features of metastases in the ovaries differ depending on the origin of the primary tumor. Ultrasound Obstet Gynecol 2007;29:505-11.

191 Ludovisi M, De Blasis I, Virgilio B, et al. Imaging in gynecological disease (9): clinical and ultrasound characteristics of tubal cancer. Ultrasound Obstet Gynecol 2014;43:328-35. 